\title{
Nova metodologia para ensino e ensaio de técnicas operatórias em cirurgia cardíaca
}

\author{
José Carlos Silva de ANDRADE*
}

ANDRADE, J. C. S. - Nova metodologia para ensino e ensaio de técnicas operatórias em cirurgia cardíaca. Rev. Bras. Cir. Cardiovasc., 9 (1): 1-21, 1994.

RESUMO: Utilizando-se os equipamentos e reproduzindo-se as instalações de um centro operatório, simulam-se todas as condiçōes para operaçōes com circulação extracorpórea (CEC). Estas são realizadas num manequim que tem peças anatômicas humanas frescas fixadas em suporte especialmente desenvolvido e colocado dentro da sua cavidade torácica. Os procedimentos são gravados em vídeo e projetados em telăo para o auditório, que mantém intercomunicação com a equipe cirúrgica. O sistema foi utilizado no XI Simpósio Internacional Unicór em julho de 1993, no $3^{2}$ Congresso da SCICVESP em novembro de 1993 e no $21^{2}$ Congresso Nacional de Cirurgia Cardiaca em março de 1994, com os seguintes procedimentos: Operação de Senning, Anastomose cavo-pulmonar, Operação de Jatene, Operação de Konno, Operação de Monaghian, Cardioplegia retrógrada, Ampliaçāo do anel aórtico com retroplegia sanguínea normotérmica contínua, Ampliação simétrica do anel aórtico (Nova proposição técnica), Plastia da valva mitral, Substituição da valva mitral, Operação do labirinto, Desfibrilador implantável e Transplante cardíaco. O método, recebido com entusiasmo pela coletividade médico-cirúrgica, mostrou ser uma eficiente técnica de ensino e de ensaio de cirurgia cardiaca, permitindo: 1) perfeita reprodução de atos operatórios em ambientes coletivos;2) excelente imagem dos procedimentos através da projeçăo de vídeo (telāo); 3) melhora na capacidade de transmissão dos ensinamentos pela clareza do campo operatório e descontração da equipe cirúrgica; 4) interrupção da "operação", sem o risco das cirurgias habituais e à qualquer momento para esclarecimentos técnicos ou táticos; 5) formação de material didático de uso permanente (videoteca) de excelente qualidade; 6 ) realização das "operaçōes" em cardiopatias congênitas ou adquiridas, através da criação de um arquivo de peças anatômicas congênitas ou adquiridas, congeladas; 7) antevisão da extensão desse procedimento a outras especialidades.

DESCRITORES: cirurgia cardíaca, técnicas operatórias; cirurgia cardíaca, métodos; cirurgia cardiaca, métodos de ensino.

\section{INTRODUÇÃO}

A história da Medicina confunde-se com a da própria humanidade. Nos seus primórdios a "arte da cura" era exercida por curandeiros ou sacerdotes, e seus ensinamentos, muitas vezes em forma de segredos ou magias, transmitidos de mestres a discípulos, em convívio diário (CONFORTI \& MAGALHÃES ${ }^{2}$, LYONS \& PETRUCELLI ${ }^{5}$, OLIVEIRA ${ }^{8}$.
A cirurgia que inicialmente aplicava-se mais a ferimentos ou traumas em guerras, foi durante a Idade Média, praticada por "cirurgiōes-barbeiros", indivíduos muito hábeis mas pouco letrados, enquanto a medicina senso geral era exercida por indivíduos mais cultos e cléricos. Henri D' Hermondaville (1260-1320) da Normandia combateu as práticas rudimentares da cirurgia entregue a ignorantes, supersticiosos e muitas vezes farsantes, 
ANDRADE, J. C. S. - Nova metodologia para ensino e ensaio de técnicas operatórias em cirurgia cardiaca. Rev. Bras. Cir. Cardiovasc., 9 (1): 1-21, 1994.

afirmando que "a cirurgia era uma tão difícil arte que apenas alguns privilegiados a poderiam alcançar". Nesta época, este mesmo autor, na Universidade de Montpellier, provavelmente pela dificuldade na conservação de peças e da exiguidade de corpos disponíveis para dissecção, foi um dos primeiros a usar figuras para o ensino da anatomia. Guy de Chauliac que com ele trabaIhou algum tempo, rebelou-se contra a utilização de quadros anatômicos que julgava não suprir o estudo direto, a seu ver imprescindivel (OLIVEIRA ${ }^{8}$ ).

A exigência do treinamento evidenciada na formação dos cirurgiões estimulou, ao longo do tempo, o emprego de animais, principalmen-

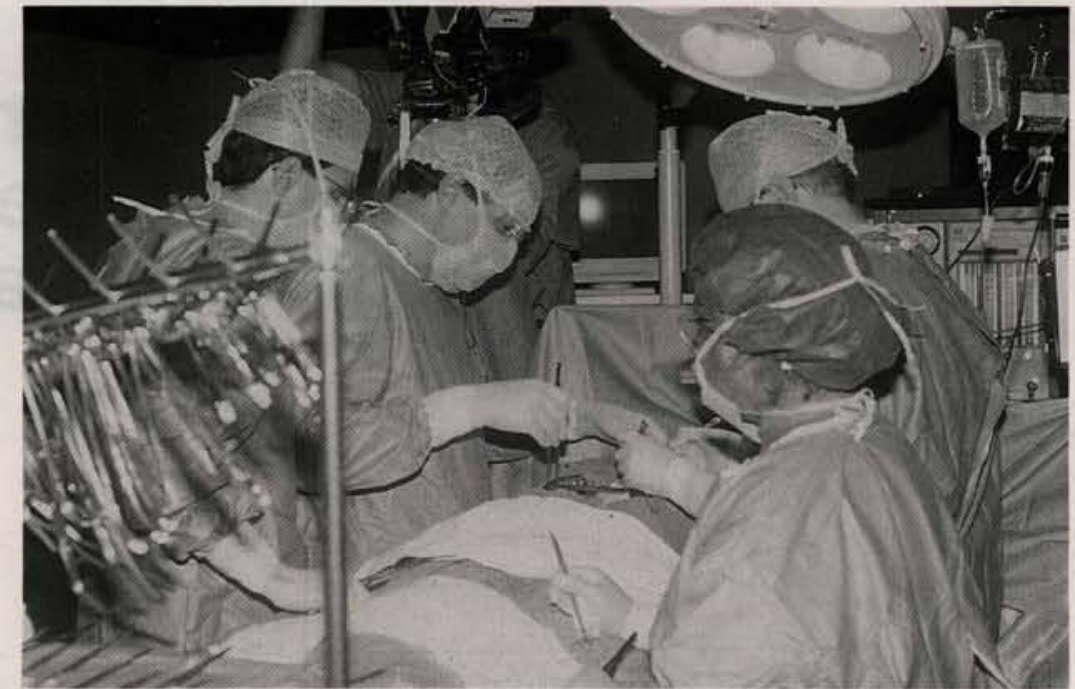

Fig. 1 - Aspecto de uma das "operações". te cães, que entretanto tinham o inconveniente de apresentar em relação ao homem importantes diferenças anatô-micas (CARREL ${ }^{1}$, MAGALHÄES ${ }^{6}$ ).

Nessa época já havia também a preocupação com a transmissão de conhecimentos cirúrgicos através de dissecções ou artigos publicados, mas em toda a evolução da medicina, e até nos dias atuais, o mais utilizado meio de formação do cirurgião tem sido o contato direto e a comunicação mestre-discípulo num convívio prolongado durante o próprio exercício profissional; convívio esse tanto mais prolongado quanto mais complexa a especialidade.

É curioso que, apesar do progresso dos métodos audiovisuais de ensino em vários setores, a sua aplicabilidade em cirurgia só recentemente começou a ser difundida. De uma maneira geral, apesar dos avanços da ciência e da técnica, o ensino da cirurgia sofreu poucas modificações e ainda hoje é feito quase que exclusivamente do contato direto mestre-discípulo no exercício contínuo da mesma com os pacientes.

Diferentes técnicas operatórias podem ser ensaiadas em cadáveres nas salas habituais de dissecção anatômica, porém, além do "desconforto" do ambiente, tem-se restrições quanto à disponibilidade de material e funcionários adequados, bem como da limitação no armazenamento de cadáveres "inteiros", e da óbvia restrição ao número de observadores.

Com o objetivo de sanar essas dificuldades e ao mesmo tempo apresentar técnicas operatórias de cirurgia cardiaca, em ambiente coletivo (simpósios e congressos), projetou-se com a simulação de um ambiente cirúrgico real (Figura 1) um modelo prático para servir como método de ensino e ensaio.

\section{MATERIAL E MÉTODO}

Reproduz-se uma sala operatória de Centro Cirúrgico (Figura 2) com todos seus habituais componentes e realiza-se uma operação cirúrgica (Figura 1) sobre um manequim que tem na sua caixa torácica uma abertura onde é colocado um coração, com suas principais estruturas vasculares, fixado em suporte (Figura 3) especialmente desenvolvido para isso.

O ato operatório é gravado em vídeo e transmitido simultaneamente para o plenário onde sob o comando de um comentador é discutido com o cirurgião e com os demais participantes.

\section{Sala operatória}

$\mathrm{Na}$ realização do "laboratório de cirurgia" constrói-se um ambiente em tudo semelhante a sala operatória de cirurgia cardíaca (Figuras 1 e 2). Nele estão presentes a mesa operatória, as mesas de instrumentação, os hampers, a máquina de circulação extracorpórea (CEC), o aspirador externo, o aparelho de anestesia, os monitores cardíacos, as mesas acessórias e bandejas para colocação de: frascos de soro, sangue, fios de sutura, drenos, equipos, esparadrapo, desinfetante, compressas, gases, material de intubação, campos cirúrgicos, material de CEC, tubos, cânulas, etc. conforme Quadros 1,2 e 3. 
ANDRADE, J. C. S. - Nova metodologia para ensino e ensaio de técnicas operatórias em cirurgia cardíaca. Rev. Bras. Cir. Cardiovasc., 9 (1): 1-21, 1994.

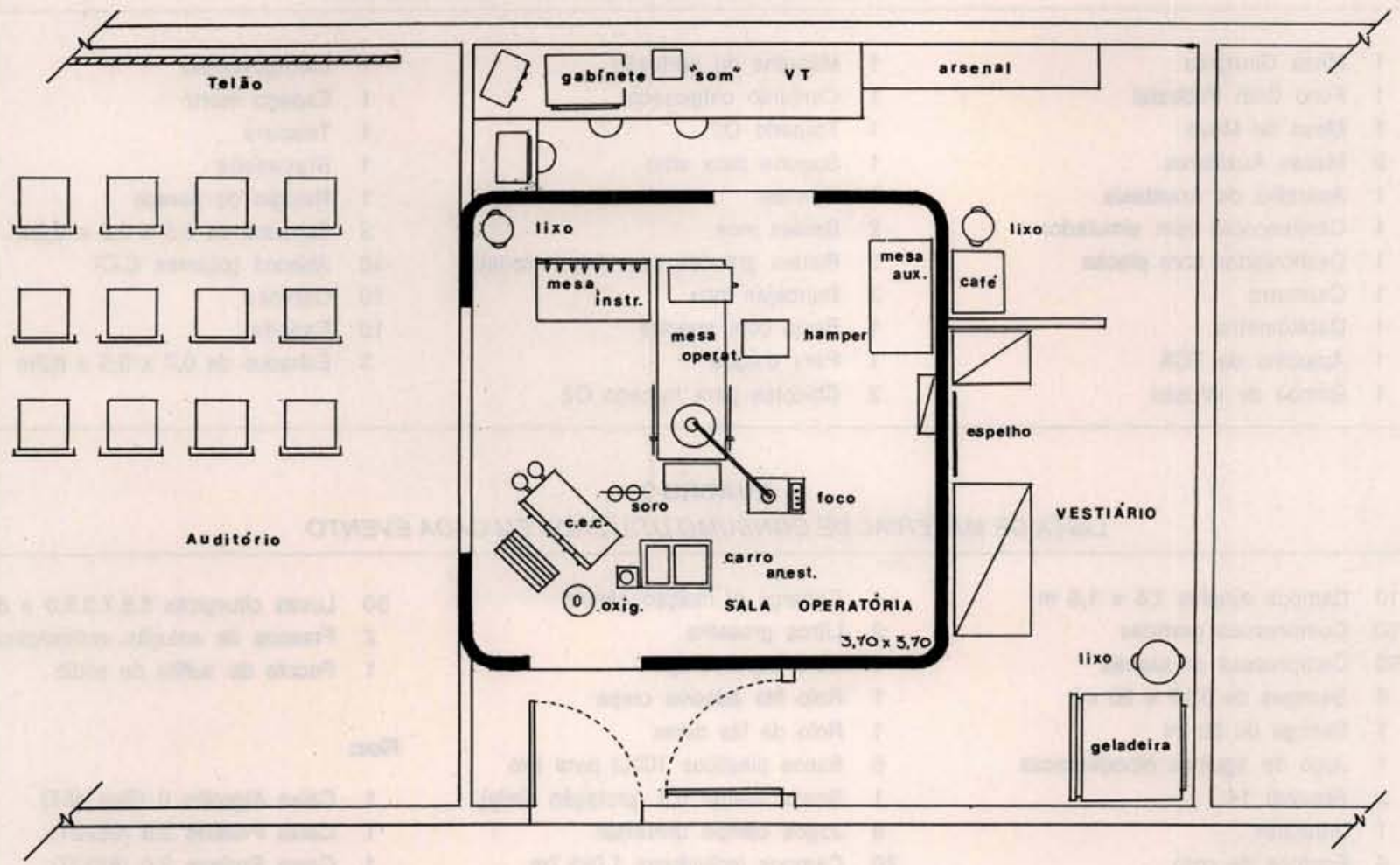

Fig. 2 - Planta fisica do Laboratório de Cirurgia

QUADRO 1

LISTA DE INSTRUMENTAL CIRÚRGICO

1 Cabo de bisturi n4

1 Cabo de bisturi $n 7$

4 Pinças vasculares $24 \mathrm{~cm}$

4 Pinças vasculares $30 \mathrm{~cm}$

2 Pinças dissecçăo

2 Pinças dente de rato

2 Pinças para tubos

4 Pinças Diethrich $18 \mathrm{~cm}$

4 Pinças Rochester

4 Pinças Kocher $14 \mathrm{~cm}$

1 Pinça Mixter $18 \mathrm{~cm}$

1 Pinça Mixter $22 \mathrm{~cm}$

2 Pinças Moynihan $19 \mathrm{~cm}$

12 Pinças Kelly $14 \mathrm{~cm}$

12 Pinças Backhaus $15 \mathrm{~cm}$

25 Pinças Halstead mosquito $12 \mathrm{~cm}$

4 Porta-agulhas Hegar
2 Porta-agulhas Diethrich $18 \mathrm{~cm}$

2 Porta-agulhas Diethrich $21 \mathrm{~cm}$

3 Porta-agulhas ponta de vídea $18 \mathrm{~cm}$

3 Porta-agulhas ponta de vídea $21 \mathrm{~cm}$

1 Pinça Satinsky grande

1 Pinça Satinsky médio

1 Pinça Satinsky pequeno

1 Afastador de Ankeney

1 Pinça de aorta DeBakey

1 Pinça Derra pequeno

2 Pinças Duval

1 Tesoura Mayo curva

1 Tesoura Mayo reta

2 Tesoura Metzenbaum 18

1 Tesoura Metzenbaum 20

1 Tesoura Metzenbaum 24

1 Tesoura Diethrich 25
1 Tesoura Diethrich 45

1 Tesoura Diethrich 60

1 Tesoura Diethrich 130

1 Régua metálica de $20 \mathrm{~cm}$

1 Jogo de velas Hegar

3 Pinças de simpatectomia

1 Jogo de afastador para aorta

1 Jogo de afastador para atrio

1 Pinça para valva mitral

1 Saca trombo

1 Bisturi rombo

1 Aspirador reto

1 Aspirador angulado

1 y de $1 / 4^{\prime \prime} x 1 / 4^{\prime \prime} \times 1 / 2 "$

1 Conexão $3 / 8^{\prime \prime} \times 1 / 4^{\prime \prime}$ 
ANDRADE, J. C. S. - Nova metodologia para ensino e ensaio de técnicas operatórias em cirurgia cardiaca. Rev. Bras. Cir. Cardiovasc., 9 (1): 1-21, 1994.

\section{QUADRO 2}

1 Mesa Cirúrgica

1 Foco Com Pedestal

1 Mesa de Mayo

2 Mesas Auxiliares

1 Aparelho de Anestesia

1 Cardioscópio com simulador

1 Desfibrilador com placas

1 Oximetro

1 Debitômetro

1 Aparelho de TCA

1 Bomba de infusão
1 Máquina de perfusāo

1 Conjunto oxigenador

1 Torpedo $\mathrm{O} 2$

1 Suporte para soro

1 Hamper

2 Baldes inox

3 Baldes grandes para lixo hospitalar

3 Bandejas inox

1 Bacia com suporte

1 Pera d'água

2 Chicotes para torpedo $\mathrm{O} 2$
1 Laringoscópio

1 Espaço morto

1 Tesoura

1 Braçadeira

1 Relógio de parede

3 Estrados de $0,5 \times 0,3 \times 0,2 \mathrm{~m}$

30 Jalecos (pijamas C.C)

10 Cabides

10 Espelho

3 Estrados de $0,7 \times 0,5 \times 0,2 \mathrm{~m}$

\section{QUADRO 3}

LISTA DE MATERIAL DE CONSUMO UTILIZADA EM CADA EVENTO

\begin{tabular}{|c|c|c|c|c|c|}
\hline 10 & Campos simples $1,6 \times 1,6 \mathrm{~m}$ & 1 & Cadarço p/ fixaçāo cânula & 50 & Luvas cirurgicas $6 \cdot 5,7.0,8.0$ \\
\hline 100 & Compressas grandes & 2 & Litros groselha & 2 & Frascos de solução antisséptice \\
\hline 50 & Compressas pequenas & 1 & Rolo esparadrapo & 1 & Pacote de sulfito de sódio \\
\hline 6 & Seringas de 5,10 e $20 \mathrm{ml}$ & 1 & Rolo fita adesiva crepe & & \\
\hline 1 & Seringa de $50 \mathrm{ml}$ & 1 & Rolo de fita durex & & \\
\hline 1 & Jogo de agulhas hipodérmicas & 6 & Sacos plasticos $100 \mathrm{Lt}$ para lixo & Fios: & \\
\hline 2 & Abocath 14 & 1 & Sonda uretral n. 8 (proteção Kelly) & 1 & Caixa Algodāo 0 (Sap 45T) \\
\hline 1 & Intracath & 6 & Jogos campo universal & 1 & Caixa Prolene 3.0 (9522T) \\
\hline 6 & Equipos de soro & 20 & Campos individuais $1.0 \times 1.7 \mathrm{~m}$ & 1 & Caixa Prolene $3.0(8822 \mathrm{~T})$ \\
\hline 3 & Equipos de sangue & 5 & Campos mesa auxiliar $1.1 \times 2.1 \mathrm{~m}$ & 1 & Caixa Prolene 4.0 (9557T) \\
\hline 3 & Bolsas plásticas para sangue & 3 & Campos para mesa Mayo & 1 & Caixa Prolene 4.0 (8356T) \\
\hline 4 & Frascos de soro Ringer & 30 & Aventais & 1 & Caixa Prolene 4.0 (9521T) \\
\hline 2 & Frascos de soro fisiológico & 1 & Pacote máscara & 1 & Caixa Prolene 5.0 (9556T) \\
\hline 2 & Frascos vazios (p/cortantes) & 1 & Pacote gorro masculino & 1 & Caixa Prolene 6.0 (M8306T) \\
\hline 1 & Jogo de agulhas de sutura & 1 & Pacote tôca feminina & 1 & Caixa Ethibond 2.0 (B553T) \\
\hline 6 & Lâminas 11 & 1 & Pacote gazes & 1 & Caixa Ethibond 2.0 (BP93T) \\
\hline 6 & Lâminas 22 & 30 & Pro-pés & 1 & Kit Ethibond HKV $15 \mathrm{~g}$ \\
\hline 1 & Cânula endotraqueal c/balonete & 1 & Caixa luvas de procedimento & 1 & Caixa fita cardiaca \\
\hline
\end{tabular}

\section{Suporte}

É constituído por uma peça de madeira de $22 \times 30 \times 3 \mathrm{~cm}$ com nove pequenos orifícios $(2 \mathrm{~mm}$ de diâmetro) para introdução e fixação das extremidades dos arames de suporte (Figura 3). Estes são fios de alumínio de $2 \mathrm{~mm}$ de diâmetro (habitualmente usados em cercas rurais) sendo: 2 deles (A) em formato de alça (com dimensão de $15 \times 25 \times 15 \mathrm{~cm}$ ) colocados lateralmente (esquerda e direita) na base de madeira e fixados por ambas extremidades: 4 (B, C, D e E) de $18 \mathrm{~cm}$ de comprimento fixos numa extremidade e com uma peça plástica na outra, colocados 3 (B, C e D) cranealmente e 1 (E) caudalmente e, 1 (F) de $3 \mathrm{~cm}$ de comprimento que fixado centralmente na base de madeira tem sua extremidade livre moldada em forma de aro de

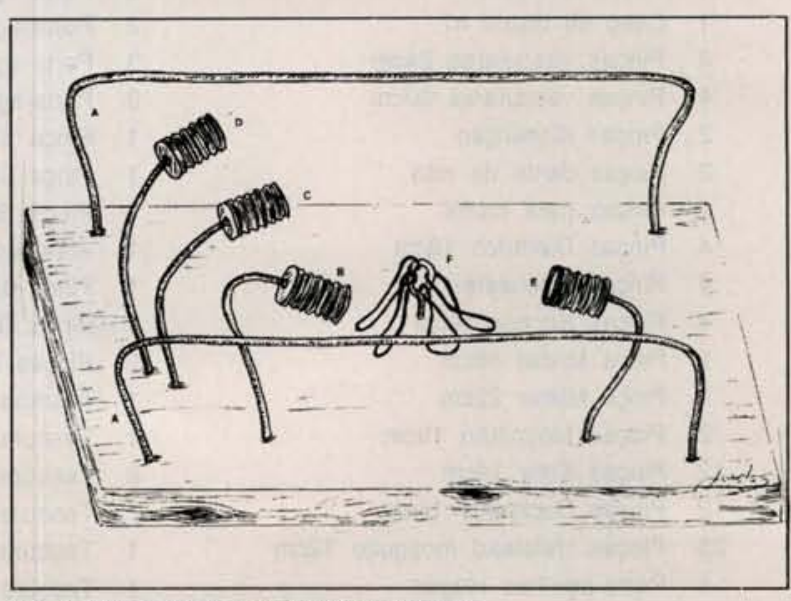

Fig. 3 - Desenho esquemático do suporte 
ANDRADE, J. C. S. - Nova metodologia para ensino e ensaio de técnicas operatórias em cirurgia cardíaca. Rev. Bras. Cir. Cardiovasc., 9 (1): 1-21, 1994.

aproximadamente $1 \mathrm{~cm}$ de diâmetro aprisionando 4 anéis elásticos (dos utilizados usualmente em bancos para prender maços de cédulas).

Os 2 arames em forma de alça colocados lateralmente tem a função de servir de suporte a um lenço de borracha $(0,6 \mathrm{~mm}$ de espessura) de $40 \times 30$ $\mathrm{cm}$ sobre o qual é posto o coração, e também, para a amarração dos fios de tração das bordas pericárdicas. Posteriormente, após toda a fixação do coração ao suporte e colocação dos campos operatórios, sobre eles ficará também o Afastador de Ankeney.

Os 4 arames de $18 \mathrm{~cm}$ de comprimento se destinam à fixação das veias cavas superior $(B)$ e inferior (E), da artéria pulmonar (D) e da aorta (C). Para isso, têm na sua extremidade livre um segmento de $5 \mathrm{~cm}$ de um tubo plástico de $1 / 2$ polegada de diâmetro e com uma superfície externa corrugada para facilitar a amarração dos vasos.

$\mathrm{O}$ arame restante (F) com extremidade em anel (e com os 4 elásticos) se destina à fixação das veias pulmonares, cada uma delas tracionada por um dos elásticos.

\section{Montagem da peça anatômica no suporte}

Obtida a peça anatômica por secção distal de todos seus vasos: aorta acima do tronco braquio cefálico, art. pulmonar após sua bifurcação, veia cava inferior abaixo do diafragma, veia cava superior acima da desembocadura da veia ázigos, e veias pulmonares o mais longe possível do atrio esquerdo, ela é exaustivamente lavada: introduz-se água corrente através dos vários vasos e massageiase o coração procurando-se retirar todos eventuais coágulos formados no seu interior.

O pericárdio é aberto longitudinalmente e o coração disposto sobre o lenço de borracha já provisoriamente preso ao suporte por pinças de Backhaus. Através de pontos passados em suas bordas, fixa-se o pericárdio e simul-tâneamente o lenço de borracha às barras laterais do suporte.

Nas projeções da aorta, art. pulmonar e veias cavas retiram-se segmentos circulares do lenço de borracha, pelos quais passam-se esses vasos. Os mesmos são entāo, um por um fixados aos respectivos suportes por ligaduras com fio de algodão grosso.

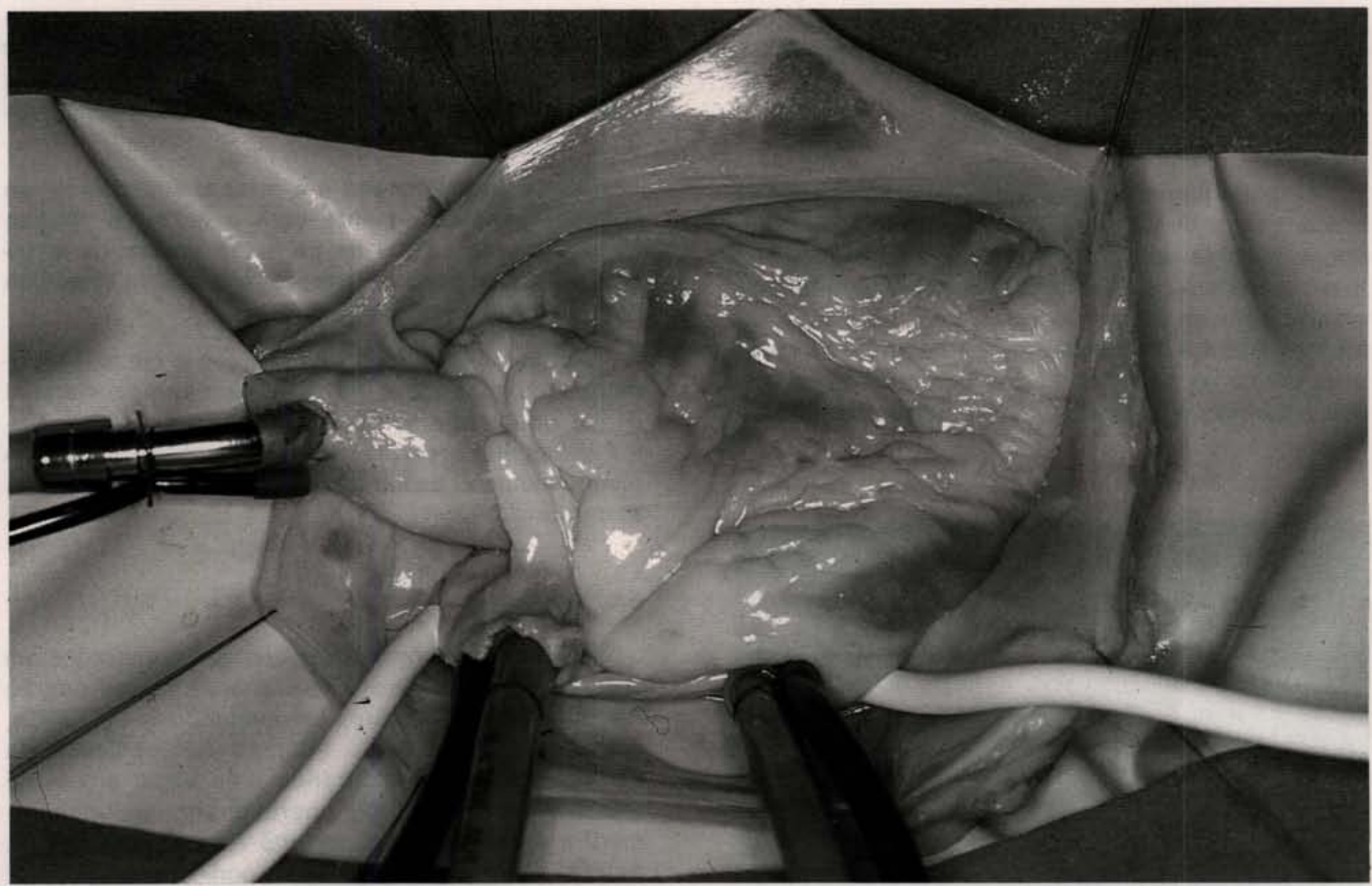

Flg. 4-Aspecto da peça montada no suporte 
ANDRADE, J. C. S. - Nova metodologia para ensino e ensaio de técnicas operatórias em cirurgia cardiaca. Rev. Bras. Cir. Cardiovasc., 9 (1): 1-21, 1994.

Cada uma das veias pulmonares é transfixada e ligada com fio de mersilene 2.0; na projeção de cada uma delas no lenço de borracha, os fios são passados através do lenço e fixados nos elásticos da base do suporte ( $F$ da Figura 3 ).

As veias cavas são dissecadas e reparadas por fitas cardíacas passadas em torniquetes. Através de suturas em bolsa realizadas no atrio direito ou nas próprias veias cavas, estas são então cateterizadas com cânulas de tubo plástico transparente de $1 / 4$ de polegada de diâmetro, previamente pintados pela sua face interna com tinta esmalte sintética de cor vermelho escura. As cânulas são fixadas aos torniquetes das suturas em bolsa por ligaduras com algodão grosso (Figura 4).

A aorta também é cateterizada através de sutura em bolsa realizada na sua face anterior. Utiliza-se cateter metálico com extensão de tubo plástico ou cateter plástico específico, ambos pintados pela sua face interna de vermelho rutilante, e faz-se sua fixação ao torniquete da sutura em bolsa.

\section{Conservação}

Toda a superfície exposta da peça é recoberta com filme transparente de PVC "Magipach" e o conjunto envolvido por campo operatório é embalado em saco plástico, identificado e acondicionado em congelador à -16 graus centígrados.

\section{Manequim}

O manequim que apresenta cabeça, pescoço e braço, com mão de látex, tem seu tórax constituído por uma caixa de madeira em semi-arco (como se fosse a metade de um tonel cortado longitudinalmente) com altura de $20 \mathrm{~cm}$ e com uma abertura na face anterior de $25 \times 35 \mathrm{~cm}$; o abdome e os membros inferiores são delineados por campos moldados seguindo o formato dessas partes do corpo.

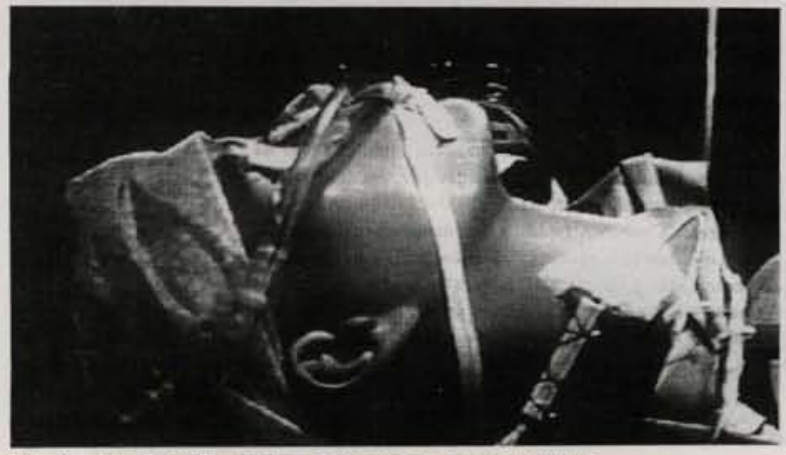

Fig. 5 - Aspecto da cabeça e pescoço do manequim.
$\mathrm{Na}$ cabeça (Figura 5), vedam-se os olhos, com pequenas tiras de esparadrapo e introduz-se parte de canula endotraqueal na boca completando-se sua fixação com cadarço. Um intra-cath é fixado no pescoço, como se estivesse em veia jugular, e feita conexão com torneira de 3 vias à bolsa de "sangue" (bolsa com soro tingido de cor vermelha por anilina ou groselha) que goteja continuamente, recolhendo-se esse líquido em outra bolsa sob a mesa. De sob o tórax saem também os fios que fazem conexão com o monitor de ritmo cardíaco (cardioscópio).

No braço, fixado na braçadeira simula-se a cateterização da artéria radial e faz-se a devida conexão com o manômetro de pressão arterial.

\section{Montagem do suporte no manequim}

O suporte (já com o coração devidamente montado e com as canulas arterial e venosas colocadas) é retirado do congelador 12 horas antes do procedimento, para uma eficiente descongelação. Sobre toda a superfície da peça anatômica é aspergida uma solução de sulfito de sódio a $2 \%$ que tem o poder de reavivar sua coloração.

O suporte é colocado no interior da "cavidade torácica". O manequim recebe então a cobertura dos campos operatórios e delimita-se a abertura torácica com campos menores fazendo-se com que todas as partes do suporte fiquem inaparentes. 0 afastador de Ankeney é colocado apoiado sobre a superfície do tórax e parte sobre as abas do suporte.

As cânulas venosas e a arterial são postas em conexão respectivamente com a linha de retorno venoso e com a linha de perfusão arterial do sistema de CEC, pintadas respectivamente de vermelho escuro e vermelho rutilante.

Aproxima-se a mesa de Mayo e a mesa instrumental habitual sobre as quais ordena-se o material citado no Quadro 1. Colocam-se no campo as pontas de aspiradores (interno e externo) e o cabo do bisturi elétrico, deixando-se o sistema pronto para a demonstração.

\section{Audiovisua}

Os procedimentos cirúrgicos são registrados em vídeo empregando-se três câmeras, todas padrão "broadcasting". Da marca lkegami modelo ITC $730 \mathrm{~A}$, uma delas é fixada ao teto, na perpendicular do campo operatório, mostrando uma visão ampla do mesmo. Uma segunda, operada por um "cameramen" e apoiada sobre um tripé é localizada atrás e ao lado do cirurgião; com um "zoom" de $36 x$ permite a tomada de cenas com grande aproximação, for- 
ANDRADE, J. C. S. - Nova metodologia para ensino e ensaio de técnicas operatórias em cirurgia cardiaca. Rev. Bras. Cir. Cardiovasc., 9 (1): 1-21, 1994.

necendo detalhes do ato operatório (Figura 6). Uma terceira, também operada por "camera-men", é disposta no auditório para focalização do coordenador e da platéia.

Todas as câmeras tem suas imagens monitorizadas na cabine de audio e VT, e controladas por um "Switcher" processado por um computador IBM-PC 486. Auxiliado pelo Diretor Científico (médico coordenador), o Diretor de Vídeo, que também processa os microfones (do cirurgião, do comentador e da platéia), realiza os cortes de imagem. As imagens selecionadas são transmitidas ao "telão" localizado no auditório através de um Projetor de Vídeo Sony 1041, com placa controladora de sinal VM-Studio, da Fast.

Automaticamente, toda a projeção é gravada em vídeo, no padrão U-Matic, para posterior edição e integração à videoteca.

\section{Método de avaliação}

\section{Qualitativo}

Todos os participantes ativos (cirurgiões e comentadores) dos Laboratórios de Cirurgia efetuados, manifestaram sua opinião sobre a Nova metodologia para ensino e ensaio de técnicas operatórias em cirurgia cardíaca. Essas manifestações foram reproduzidas integralmente na seção Depoimentos do capítulo: Resultados.

\section{Quantitativo}

Através de notas de avaliação (ótimo, bom, regular, ruim) foram estudados os seguintes quesitos do método de ensino proposto: 1-capacidade de simulação do ato operatório, 2-qualidade do áudio, 3-qualidade do visual, 4-capacidade didática, 5-capacidade de adestramento técnico, 6-aplicabilidade em eventos científicos, 7-aplicabilidade em escolas médicas (para demonstrações e ensino de técnicas operatórias - curso de graduação), 8-aplicabilidade para alunos, residentes, estagiá-rios de cirurgia cardíaca (ensino e padronização dos tempos operatórios), 9-aplicabilidade para profissionais, especialistas em cirurgia cardíaca (apresentação de novas técnicas ou ensaios de variações técnicas).

As notas de avaliação obtidas através do preenchimento de questionário (Quadro 4) foram dadas por três grupos de avaliadores: Grupo A- Cirurgiões cardiovasculares com participação ativa no Laboratório (cirurgiões ou comentadores), Grupo P. Cirurgiőes cardiovasculares com participação passiva (assistentes) no Laboratório, e Grupo G- Cirurgiōes de outras especialidades (técnica operatória), que assistiram durante o Curso de Pós-Graduação em Técnica operatória e Cirurgia Experimental da Escola Paulista de Medicina à exposição explicativa do método e a segmentos dos "video tapes" realizados durante os Laboratórios de Cirurgia.

O Grupo A foi integrado por 23 cirurgiões, o P por 30 e o G por 40 , obtendo-se portanto para os 9 quesitos formulados: 207 respostas no Grupo A, 270 no Grupo P e 360 no Grupo G num total de 837 respostas.

Essas respostas foram tabuladas e apresentadas na seção Tabelas do capítulo RESULTADOS. 


\section{FICHA DE AVALIAÇÃo}

TRABALHO: NOVA METODOLOGIA PARA ENSINO E ENSAIO DE TÉCNICAS OPERATÓRIAS EM CIRURGIA CARDÍACA

AVALIADOR:

I. CAPACIDADE DE SIMULAÇÃO DO ATO OPERATÓRIO:
$\square$ ÓTIMO
$\square$ BOM
$\square$ REGULAR
$\square$ RUIM

II. QUALIDADE DO ÁUDIO:
$\square$ ÓTIMO
$\square$ BOM
GEGULAR
D RUIM

III. QUALIDADE DO VISUAL:
口 ÓTIMO
$\square \mathrm{BOM}$
REGULAR
$\square$ RUIM

IV. CAPACIDADE DIDÁTICA, PODER DE TRANSMISSÃO DE ENSINAMENTOS:
口 ÓTIMO
$\square$ BOM
$\square$ REGULAR
D RUIM

V. CAPACIDADE DE ADESTRAMENTO TÉCNICO, UTILIZANDO O MODELO PARA TREINAMENTO CIRÚRGICO:

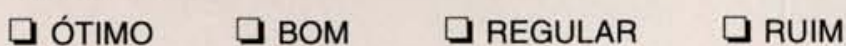

VI. APLICABILIDADE EM EVENTOS CIENTIFICOS (CONGRESSOS, SIMPÓSIOS ETC. DA ESPECIALIDADE: $\square$ ÓTIMO $\square$ BOM $\square$ REGULAR $\square$ RUIM

VII. APLICABILIDADE EM ESCOLAS MÉDICAS (PARA DEMONSTRAÇÕES E ENSINO DA TÉCNICA OPERATÓRIA)

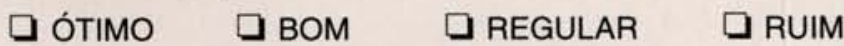

VIII. APLICABILIDADE PARA ALUNOS DE CIRURGIA CARDÍACA (PARA ENSINO E PADRONIZAÇÃO DOS TEMPOS OPERATÓRIOS):

$\square$ ÓTIMO $\square$ BOM $\square$ REGULAR $\square$ RUIM

IX. APLICABILIDADE PARA ESPECIALISTAS EM CIRURGIA CARDÍACA (PARA APRESENTAÇÃO DE NOVAS TÉCNICAS OU ENSAIOS DE VARIAÇÕES TÉCNICAS):

$\square$ ÓTIMO $\square$ BOM $\square$ REGULAR $\square$ RUIM 
ANDRADE, J. C. S. - Nova metodologia para ensino e ensaio de técnicas operatórias em cirurgia cardiaca. Rev. Bras. Cir. Cardiovasc., 9 (1): 1-21, 1994.

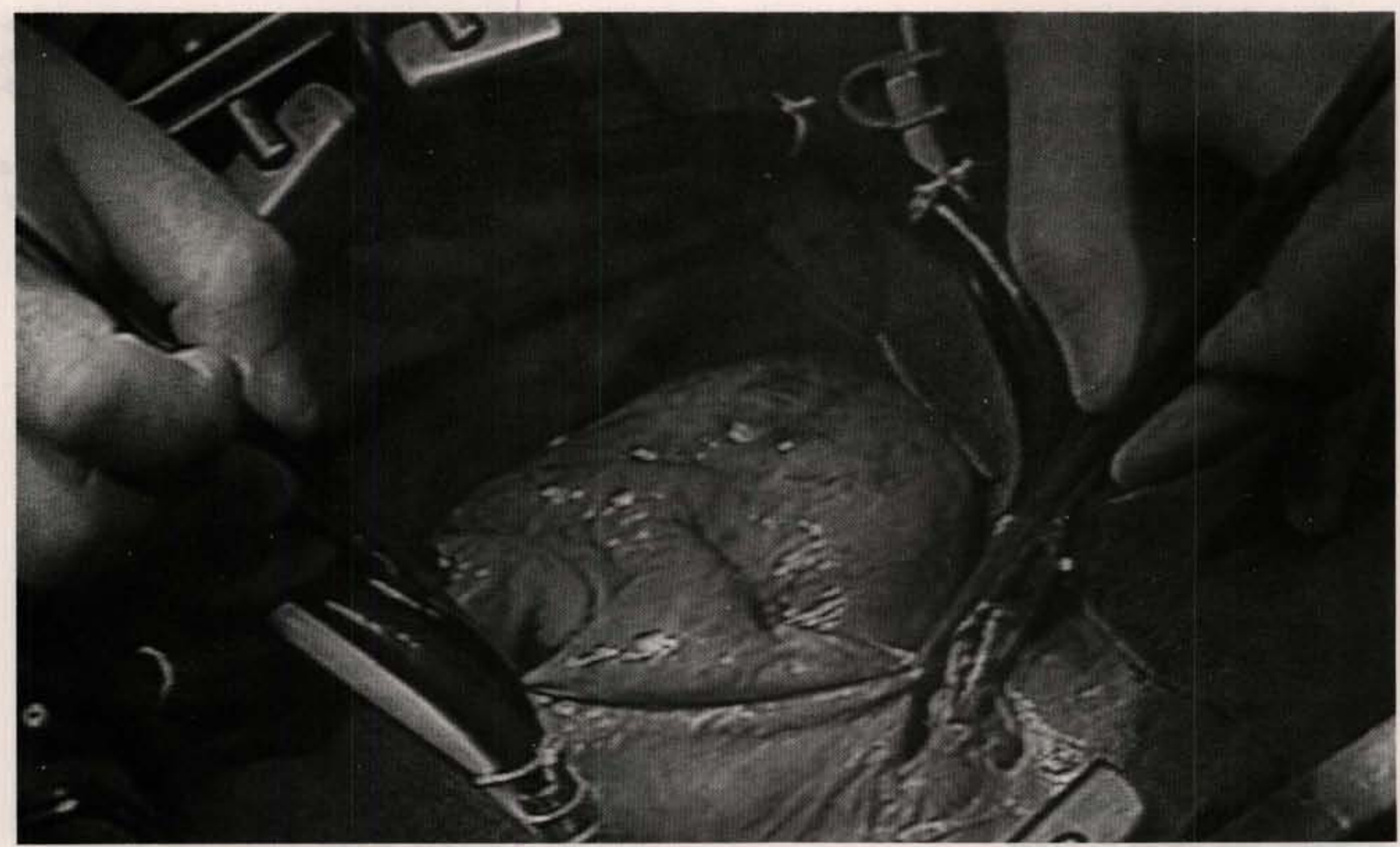

Fig. 6 - Imagem observada no "telāo",

\section{Método estatístico}

Utilizou-se o Teste do Qui-quadrado para Tabelas RxS (SIEGEL, ${ }^{7}$ ) com o objetivo de comparar, para o total das respostas, os grupos de cirurgiões: cardíaco ativo, cardíaco passivo e geral, em relação às distribuições percentuais de respostas: ótimo, bom, regular e ruim.

Fixou-se em 0.05 ou $5 \%(\alpha=0.05)$ o nível de rejeição da hipótese de nulidade, assinalando-se com um asterisco os valores significantes.

\section{RESULTADOS}

Apresentado pela primeira vez no XI Simpósio Internacional Unicór, em 23 de julho de 1993, no Hotel Maksoud Plaza em São Paulo, o Laboratório de Cirurgia Cardíaca permitiu a realização e discussão de seis procedimentos: Operação de Senning, Ampliação do anel aórtico, Plastia da valva mitral, Substituição da valva mitral, Operação do "labirinto" e Transplante cardiaco. Essas demonstrações, gravadas e exibidas simultaneamente em telão foram realizadas por escolhidos e competentes cirurgiões habituados ao ensino da especialidade, que durante o ato operatório debatiam com a platéia, explicavam, repetiam determinados detalhes técnicos sob a coordenação de outros experientes profissionais, conforme se observa no Quadro 5.

Em 26 e 27 de novembro de 1993 o Laboratório de Cirurgia Cardíaca foi levado a efeito durante o $3^{2}$ Congresso da Sociedade de Cirurgia Cardiovascular do Estado de São Paulo (SCICVESP), realizado na Sociedade de Medicina e Cirurgia da cidade de São José do Rio Preto, seguindo os mesmos principios utilizados. Efetuaram-se os seguintes procedimentos: Operação de Senning, Operação de Konno, Operação da fibrilação atrial, Plastia mitral com cardioplegia retrógrada, Desfibrilador implantavel e Transplante cardíaco, conforme Quadro 6.

Em 24 de março de 1994, por ocasião do $21^{\circ}$ Congresso Nacional de Cirurgia Cardíaca, o Laboratório de Cirurgia foi realizado no Centro de Eventos São José do Hotel Plaza São Rafael, em Porto Alegre. Foram efetuados, conforme se observa no Quadro 7, seis procedimentos: Cirurgia do "labirinto" na fibrilação atrial, Anastomose cavo-pulmonar, Ampliação do anel aórtico com retroplegia sanguínea normotérmica contínua, Ampliação simétrica do anel aórtico (nova proposição técnica), Translocação das artérias coronárias e dos grandes vasos da base na Transposição dos grandes vasos da base, e Transplante cardíaco ortotópico. 
Em todos os eventos, as "operações" reproduziram quase fielmente as condições habituais. Sua projeção em telão ofereceu imagem nítida e detaIhada das várias técnicas apresentadas.

A possibilidade da interrupção da "operação", a qualquer instante e sem nenhum risco, incentivou a participação da platéia que, interagindo com o cirur- gião, solicitava esclarecimentos sobre a técnica, repetição de manobras, ou mesmo questionava determinados detalhes técnicos ou táticos, sugerindo algumas alternativas.

A gravação com registro em fita de vídeo das "operações" já permitiu a formação de uma pequena videoteca com 18 fitas.

\section{QUADRO 5}

\section{LABORATÓRIO DE CIRURGIA CARDÍACA \\ XI SIMPÓSIO INTERNACIONAL UNICÓR - SÃO PAULO - SP.}

23 de julho de 1993

8:30 - 9:30 h

Operação de Senning

Cirurgião: Miguel A. Maluf

Comentador: Domingos M. Braile

9:45 - 10:45 h

Ampliação do anel aórtico

Cirurgião: Luiz Carlos B. Souza

Comentador: Domingos M. Braile

11:00 - 12:00 h

Prótese mitral sem suporte

Cirurgião: Bayard Gontijo Filho

Comentador: Domingos M. Braile
$14: 30-15: 30 \mathrm{~h}$
Cirurgia da fibrilação atrial
Cirurgião: Marcelo Jatene
Comentador: José Wanderley Netto
$15: 45-16: 45 \mathrm{~h}$
Plastia da valva mitral
Cirurgião: Francisco Gregori Jr.
Comentador: José Wanderley Netto
17:00 - 18:00 h
Transplante cardíaco
Cirurgião: João Nelson R. Branco
Comentador: José Wanderley Netto

\section{LABORATÓRIO DE CIRURGIA CARDÍACA $3^{\circ}$ CONGRESSO DA SOCIEDADE DE CIRURGIA CARDIOVASCULAR DO ESTADO DE SÃO PAULO SÃO JOSÉ DO RIO PRETO - SP.}

26 de novembro de 1993

$8: 30$ - 9:30 h

Operação de Senning

Cirurgião: Miguel A. Maluf

Comentador: Ricardo Sgarbieri

10:00 - 11:00 h

Operação de Konno

Cirurgião: Luiz Carlos Bento de Souza

Comentador: Luiz Antonio Rivetti

$11: 30-12: 30 \mathrm{~h}$

Operação da fibrilação atrial

Cirurgião: Marcelo Jatene

Comentador: Jarbas J. Dinkhuysen
27 de novembro de 1993

$8: 30-9: 30 \mathrm{~h}$

Plastia mitral com cardioplegia retrógada

Cirurgião: Domingos M. Braile

Comentador: Pablo M. A. Pomerantzeff

10:00 - 11:00 $\mathrm{h}$

Desfibrilador implantável

Cirurgião: Max Schaldach

Comentador: Decio S. Kormann

$11: 30-12: 30 \mathrm{~h}$

Transplante cardíaco

Cirurgião: João Nelson R. Branco

Comentador: Fabio Jatene 
ANDRADE, J. C. S. - Nova metodologia para ensino e ensaio de técnicas operatórias em cirurgia cardiaca. Rev. Bras. Cir. Cardiovasc., 9 (1): 1-21, 1994.

\section{LABORATÓRIO DE CIRURGIA CARDÍACA \\ $21^{\circ}$ CONGRESSO NACIONAL DE CIRURGIA CARDÍACA-PORTO ALEGRE-RS}

24 de março de 1994

$10: 30-14: 10 \mathrm{~h}$

Cirurgia do labirinto na fibrilação atrial

Cirurgião: Marcelo B. Jatene

Comentador: Francisco Gregori Jr.

$10: 30-14: 10 \mathrm{~h}$

Anastomose cavopulmonar

Cirurgião: Paulo Paredes Paulista

Comentador: Miguel Barbero Marcial

13:00 - 14:10 h

Ampliação do anel aórtico com retroplegia sanguínea normotérmica contínua.

Cirurgiāo: Domingo M. Braile

Comentador: Luiz Antonio Rivetti
$14: 20-15: 30 \mathrm{~h}$

Ampliação simétrica do anel aórtico. Nova proposição técnica.

Cirurgião: João Ricardo Santana

Comentador: Luiz Eduardo Villaça Leão

$15: 40$ - 16:50 h

Translocação das artérias coronárias e dos grandes vasos (operação de Jatene) na Transposição dos grandes vasos da base.

Cirurgião: Luiz Carlos Bento de Souza

Comentador: Danton R. da Rocha Loures

$17: 10-18: 30 \mathrm{~h}$

Transplante cardíaco ortotópico

Cirurgião: Enio Buffolo

Comentador: José Pedro da Silva

Tabelas

TABELA 1

CIRURGIŌES: CARDIACO ATIVO, CARDIACO PASSIVO E GERAL SEGUNDO AS RESPOSTAS: ÓTIMO, BOM, REGULAR E RUIM PARA O QUESITO 1: CAPACIDADE DE SIMULAÇÃO DO ATO OPERATÓRIO, NA AVALIAÇÃO DA NOVA METODOLOGIA PARA O ENSINO E ENSAIO DE TÉCNICAS OPERATÓRIAS EM CIRURGIA CARDÍACA.

\begin{tabular}{lccccccccccc}
\hline \multicolumn{1}{c}{} & \multicolumn{1}{c}{ RESPOSTAS } \\
\hline Cirurgião & \multicolumn{2}{c}{ Ótimo } & \multicolumn{2}{c}{ Bom } & \multicolumn{2}{c}{ Regular } & \multicolumn{2}{c}{ Ruim } & \multicolumn{2}{c}{ Total } \\
& $\mathrm{N}$ & $\%$ & $\mathrm{~N}$ & $\%$ & $\mathrm{~N}$ & $\%$ & $\mathrm{~N}$ & $\%$ & $\mathrm{~N}$ & $\%$ \\
\hline Ativo & 14 & 60.9 & 9 & 39.1 & 0 & 0.0 & 0 & 0.0 & 23 & 100.0 \\
Passivo & 27 & 90.0 & 3 & 10.0 & 0 & 0.0 & 0 & 0.0 & 30 & 100.0 \\
Geral & 15 & 37.5 & 25 & 62.5 & 0 & 0.0 & 0 & 0.0 & 40 & 100.0 \\
\hline Total & 56 & 60.2 & 37 & 39.8 & 0 & 0.0 & 0 & 0.0 & 93 & 100.0 \\
\hline
\end{tabular}

TABELA 2

CIRURGIÖES: CARDIACO ATIVO, CARDIACO PASSIVO E GERAL SEGUNDO AS RESPOSTAS: ÓTIMO, BOM, REGULAR E RUIM PARA O QUESITO 2: QUALIDADE DO AUDIO, NA AVALIAÇÃO DA NOVA METODOLOGIA PARA O ENSINO E ENSAIO DE TÉCNICAS OPERATÓRIAS EM CIRURGIA CARDIACA.

\begin{tabular}{|c|c|c|c|c|c|c|c|c|c|c|}
\hline \multirow[b]{3}{*}{ Cirurgião } & & & \multicolumn{6}{|c|}{ RESPOSTAS } & & \\
\hline & \multicolumn{2}{|c|}{ Ótimo } & \multicolumn{2}{|c|}{ Bom } & \multicolumn{2}{|c|}{ Regular } & \multicolumn{2}{|c|}{ Ruim } & \multicolumn{2}{|c|}{ Total } \\
\hline & $\mathrm{N}$ & $\%$ & $\mathrm{~N}$ & $\%$ & $\mathrm{~N}$ & $\%$ & $\mathrm{~N}$ & $\%$ & $\mathrm{~N}$ & $\%$ \\
\hline Ativo & 20 & 80.0 & 3 & 13.0 & 0 & 0.0 & 0 & 0.0 & 23 & 100.0 \\
\hline Passivo & 21 & 70.0 & 9 & 30.0 & 0 & 0.0 & 0 & 0.0 & 30 & 100.0 \\
\hline Geral & 20 & 50.0 & 20 & 50.0 & 0 & 0.0 & 0 & 0.0 & 40 & 100.0 \\
\hline Total & 61 & 65.6 & 32 & 34.4 & 0 & 0.0 & 0 & 0.0 & 93 & 100.0 \\
\hline
\end{tabular}


ANDRADE, J. C. S. - Nova metodologia para ensino e ensaio de técnicas operatórias em cirurgia cardíaca. Rev. Bras. Cir. Cardiovasc., 9 (1): 1-21, 1994.

TABELA 3

CIRURGIŌES: CARDIACO ATIVO, CARDIACO PASSIVO E GERAL SEGUNDO AS RESPOSTAS: ÓTIMO, BOM, REGULAR E RUIM PARA O QUESITO 3: QUALIDADE DO VISUAL, NA AVALIAÇÃO DA NOVA METODOLOGIA PARA O ENSINO E ENSAIO DE TÉCNICAS OPERATÓRIAS EM CIRURGIA CARDIACA.

\begin{tabular}{|c|c|c|c|c|c|c|c|c|c|c|}
\hline \multirow[b]{3}{*}{ Cirurgiāo } & & & \multicolumn{4}{|c|}{ RESPOSTAS } & \multirow{2}{*}{\multicolumn{2}{|c|}{ Ruim }} & \multirow{2}{*}{\multicolumn{2}{|c|}{ Total }} \\
\hline & \multicolumn{2}{|c|}{ Ótimo } & \multicolumn{2}{|c|}{ Bom } & \multicolumn{2}{|c|}{ Regular } & & & & \\
\hline & $\mathrm{N}$ & $\%$ & $\mathrm{~N}$ & $\%$ & $\mathrm{~N}$ & $\%$ & $\mathrm{~N}$ & $\%$ & $\mathrm{~N}$ & $\%$ \\
\hline Ativo & 21 & 91.3 & 2 & 8.7 & 0 & 0.0 & 0 & 0.0 & 23 & 100.0 \\
\hline Passivo & 29 & 96.7 & 1 & 3.3 & 0 & 0.0 & 0 & 0.0 & 30 & 100.0 \\
\hline Geral & 16 & 40.0 & 24 & 60.0 & 0 & 0.0 & 0 & 0.0 & 40 & 100.0 \\
\hline Total & 66 & 71.0 & 27 & 29.0 & 0 & 0.0 & 0 & 0.0 & 93 & 100.0 \\
\hline
\end{tabular}

TABELA 4

CIRURGIŌES: CARDIACCO ATIVO, CARDIACO PASSIVO E GERAL SEGUNDO AS RESPOSTAS: ÓTIMO, BOM, REGULAR E RUIM PARA O QUESITO 4: CAPACIDADE DIDÁTICA, NA AVALIAÇĀO DA NOVA METODOLOGIA PARA O ENSINO E ENSAIO DE TÉCNICAS OPERATÓRIAS EM CIRURGIA CARDÍACA.

\begin{tabular}{|c|c|c|c|c|c|c|c|c|c|c|}
\hline \multirow[b]{3}{*}{ Cirurgiāo } & \multicolumn{10}{|c|}{ RESPOSTAS } \\
\hline & \multicolumn{2}{|c|}{ Ótimo } & \multicolumn{2}{|c|}{ Bom } & \multicolumn{2}{|c|}{ Regular } & \multicolumn{2}{|c|}{ Ruim } & \multicolumn{2}{|c|}{ Total } \\
\hline & $\mathrm{N}$ & $\%$ & $\mathrm{~N}$ & $\%$ & $\mathrm{~N}$ & $\%$ & $N$ & $\%$ & $N$ & $\%$ \\
\hline Ativo & 20 & 87.0 & 3 & 13.0 & 0 & 0.0 & 0 & 0.0 & 23 & 100.0 \\
\hline Passivo & 28 & 93.3 & 2 & 6.7 & 0 & 0.0 & 0 & 0.0 & 30 & 100.0 \\
\hline Geral & 22 & 55.0 & 18 & 45.0 & 0 & 0.0 & 0 & 0.0 & 40 & 100.0 \\
\hline Total & 70 & 75.3 & 23 & 24.7 & 0 & 0.0 & 0 & 0.0 & 93 & 100.0 \\
\hline
\end{tabular}

TABELA 5

CIRURGIŌES: CARDIACO ATIVO, CARDIACO PASSIVO E GERAL SEGUNDO AS RESPOSTAS: ÓTIMO, BOM, REGULAR E RUIM PARA O QUESITO 5: CAPACIDADE DE ADESTRAMENTO TÉCNICO, UTILIZANDO O MODELO PARA TREINAMENTO CIRÚRGICO, NA AVALIAÇĀO DA NOVA METODOLOGIA PARA O ENSINO E ENSAIO DE TÉCNICAS OPERATÓRIAS EM CIRURGIA CARDIACA.

\begin{tabular}{|c|c|c|c|c|c|c|c|c|c|c|}
\hline \multicolumn{11}{|c|}{ RESPOSTAS } \\
\hline \multirow[b]{2}{*}{ Cirurgião } & \multicolumn{2}{|c|}{ Ótimo } & \multicolumn{2}{|c|}{ Bom } & \multicolumn{2}{|c|}{ Regular } & \multicolumn{2}{|c|}{ Ruim } & \multicolumn{2}{|c|}{ Total } \\
\hline & $N$ & $\%$ & $\mathrm{~N}$ & $\%$ & $\mathrm{~N}$ & $\%$ & $\mathrm{~N}$ & $\%$ & $\mathrm{~N}$ & $\%$ \\
\hline Ativo & 13 & 56.5 & 9 & 39.1 & 1 & 4.3 & 0 & 0.0 & 23 & 100.0 \\
\hline Passivo & 24 & 80.0 & 6 & 20.0 & 0 & 0.0 & 0 & 0.0 & 30 & 100.0 \\
\hline Geral & 21 & 52.5 & 19 & 47.5 & 0 & 0.0 & 0 & 0.0 & 40 & 100.0 \\
\hline Total & 58 & 73.1 & 34 & 36.6 & 1 & 4.3 & 0 & 0.0 & 93 & 100.0 \\
\hline
\end{tabular}

TABELA 6

CIRURGIÕES: CARDIACO ATIVO, CARDIACO PASSIVO E GERAL SEGUNDO AS RESPOSTAS: ÓTIMO, BOM, REGULAR E RUIM PARA O QUESITO 6: APLICABILIDADE EM EVENTOS CIENTÍFICOS (CONGRESSOS, SIMPÓSIOS, ETC.) DA ESPECIALIDADE, NA AVALIAÇÃO DA NOVA METODOLOGIA PARA O ENSINO E ENSAIO DE TÉCNICAS OPERATÓRIAS EM CIRURGIA CARDIAACA.

\begin{tabular}{|c|c|c|c|c|c|c|c|c|c|c|}
\hline \multirow[b]{3}{*}{ Cirurgiāo } & \multicolumn{8}{|c|}{ RESPOSTAS } & \multirow{2}{*}{\multicolumn{2}{|c|}{ Total }} \\
\hline & \multicolumn{2}{|c|}{ Ótimo } & \multicolumn{2}{|c|}{ Bom } & \multicolumn{2}{|c|}{ Regular } & \multicolumn{2}{|c|}{ Ruim } & & \\
\hline & $N$ & $\%$ & $\mathrm{~N}$ & $\%$ & $\mathrm{~N}$ & $\%$ & $N$ & $\%$ & $\mathrm{~N}$ & $\%$ \\
\hline$\overline{\text { Ativo }}$ & 21 & 91.3 & 2 & 8.7 & 0 & 0.0 & 0 & 0.0 & 23 & 100.0 \\
\hline Passivo & 29 & 96.7 & 1 & 3.3 & 0 & 0.0 & 0 & 0.0 & 30 & 100.0 \\
\hline Geral & 24 & 60.0 & 15 & 37.5 & 1 & 2.5 & 0 & 0.0 & 40 & 100.0 \\
\hline Total & 74 & 79.6 & 18 & 19.3 & 1 & 2.5 & 0 & 0.0 & 93 & 100.0 \\
\hline
\end{tabular}


ANDRADE, J. C. S. - Nova metodologia para ensino e ensaio de técnicas operatórias em cirurgia cardiaca. Rev. Bras. Cir. Cardiovasc., $9(1): 1-21,1994$.

\section{TABELA 7}

CIRURGIŌES: CARDIACO ATIVO, CARDIACO PASSIVO E GERAL SEGUNDO AS RESPOSTAS: ÓTIMO, BOM, REGULAR E RUIM PARA O QUESITO 7: APLICABILIDADE EM ESCOLAS MÉDICAS (PARA DEMONSTRAÇŌES E ENSINO DA TECCNICA OPERATÓRIA), NA AVALIAÇÃO DA NOVA METODOLOGIA PARA O ENSINO E ENSAIO DE TÉCNICAS OPERATÓRIAS EM CIRURGIA CARDIACA.

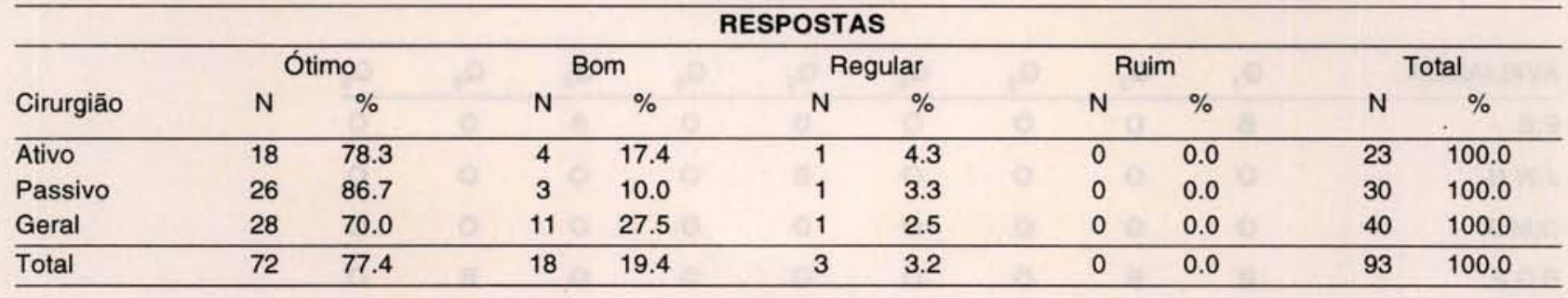

TABELA 8

CIRURGIŌES: CARDIACO ATIVO, CARDIACO PASSIVO E GERAL SEGUNDO AS RESPOSTAS: ÓTIMO, BOM, REGULAR E RUIM PARA O QUESITO 8: APLICABILIDADE PARA ALUNOS DE CIRURGIA CARDIACA (PARA ENSINO E PADRONIZAÇÃO DOS TEMPOS OPERATÓRIOS), NA AVALIAÇĀO DA NOVA METODOLOGIA PARA O ENSINO E ENSAIO DE TÉCNICAS OPERATÓRIAS EM CIRURGIA CARDÍACA.

\begin{tabular}{|c|c|c|c|c|c|c|c|c|c|c|}
\hline \multirow[b]{3}{*}{ Cirurgiāo } & & $=$ & \multicolumn{4}{|c|}{ RESPOSTAS } & \multicolumn{2}{|c|}{+2} & \multirow{2}{*}{\multicolumn{2}{|c|}{ Total }} \\
\hline & \multicolumn{2}{|c|}{ Ótimo } & \multicolumn{2}{|c|}{ Bom } & \multicolumn{2}{|c|}{ Regular } & \multicolumn{2}{|c|}{ Ruim } & & \\
\hline & $\mathrm{N}$ & $\%$ & $\mathrm{~N}$ & $\%$ & $\mathrm{~N}$ & $\%$ & $\mathrm{~N}$ & $\%$ & $\mathrm{~N}$ & $\%$ \\
\hline Ativo & 16 & 69.6 & 6 & 26.1 & 1 & 4.3 & 0 & 0.0 & 23 & 100.0 \\
\hline Passivo & 28 & 93.3 & 2 & 6.7 & 0 & 0.0 & 0 & 0.0 & 30 & 100.0 \\
\hline Geral & 27 & 67.5 & 13 & 32.5 & 0 & 0.0 & 0 & 0.0 & 40 & 100.0 \\
\hline Total & 71 & 76.3 & 21 & 22.6 & 1 & 1.1 & 0 & 0.0 & 93 & 100.0 \\
\hline
\end{tabular}

TABELA 9

CIRURGIŌES: CARDIACO ATIVO, CARDIACO PASSIVO E GERAL SEGUNDO AS RESPOSTAS: ÓTIMO, BOM, REGULAR E RUIM PARA O QUESITO 9: APLICABILIDADE PARA ESPECIALISTAS EM CIRURGIA CARDIACA (PARA APRESENTAÇĀO DE NOVAS TÉCNICAS OU ENSAIOS DE VARIAÇÕES TÉCNICAS), NA AVALIAÇÃO DA NOVA METODOLOGIA PARA O ENSINO E ENSAIO DE TÉCNICAS OPERATÓRIAS EM CIRURGIA CARDÍACA.

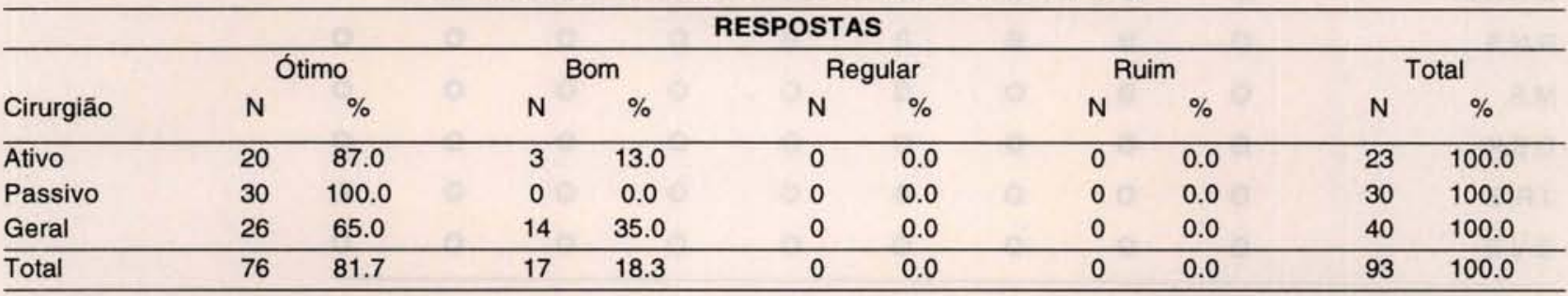

TABELA 10

CIRURGIÕES: CARDIACO ATIVO, CARDIACO PASSIVO E GERAL SEGUNDO AS RESPOSTAS: ÓTIMO, BOM, REGULAR E RUIM PARA O TOTAL DOS 9 QUESITOS DO QUESTIONÁRIO PARA AVALIAÇĀO DA NOVA METODOLOGIA PARA O ENSINO E ENSAIO DE TÉCNICAS OPERATÓRIAS EM CIRURGIA CARDIACA.

\begin{tabular}{|c|c|c|c|c|c|c|c|c|c|c|}
\hline \multirow{3}{*}{ Cirurgião } & \multicolumn{8}{|c|}{ RESPOSTAS } & & \\
\hline & \multicolumn{2}{|c|}{ Ótimo } & \multicolumn{2}{|c|}{ Bom } & \multicolumn{2}{|c|}{ Regular } & \multicolumn{2}{|c|}{ Ruim } & \multicolumn{2}{|c|}{ Total } \\
\hline & $\mathrm{N}$ & $\%$ & $\mathrm{~N}$ & $\%$ & $\mathrm{~N}$ & $\%$ & $\mathrm{~N}$ & $\%$ & $\mathrm{~N}$ & $\%$ \\
\hline Ativo & 20 & 87.0 & 3 & 13.0 & 0 & 0.0 & 0 & 0.0 & 23 & 100.0 \\
\hline Ativo & 163 & 78.7 & 41 & 19.3 & 3 & 1.5 & 0 & 0.0 & 207 & 100.0 \\
\hline Passivo & 242 & 89.6 & 27 & 10.0 & 1 & 0.4 & 0 & 0.0 & 270 & 100.0 \\
\hline Geral & 199 & 55.3 & 159 & 44.2 & 2 & 0.5 & 0 & 0.0 & 360 & 100.0 \\
\hline Total & 604 & 72.2 & 227 & 27.1 & 6 & 0.7 & 0 & 0.0 & 837 & 100.0 \\
\hline
\end{tabular}

Teste do qui quadrado: $\quad \mathrm{X}^{2}$ calculado: 100.86 * 
ANDRADE, J. C. S. - Nova metodologia para ensino e ensaio de técnicas operatórias em cirurgia cardiaca. Rev. Bras. Cir. Cardiovasc., 9 (1): 1-21, 1994.

TABELA 11

RESPOSTAS: O(ÓTIMO), B(BOM), R(REGULAR) E R(RUIM) PARA OS 9 QUESITOS FORMULADOS (Q, A Q⿻) SEGUNDO O GRUPO A CONSTITUÍDO POR 23 CIRURGIÖES CARDIOVASCULARES COM PARTICIPAÇÃO ATIVA (CIRURGIÓES OU COMENTADORES) NO LABORATÓRIO DE CIRURGIA.

\begin{tabular}{|c|c|c|c|c|c|c|c|c|c|}
\hline AVALIADOR & $Q_{1}$ & $Q_{2}$ & $Q_{3}$ & $Q_{4}$ & $Q_{5}$ & $Q_{6}$ & $Q_{7}$ & $Q_{8}$ & $Q_{9}$ \\
\hline E.B. & B & 0 & 0 & 0 & B & 0 & B & 0 & 0 \\
\hline J.W.N. & 0 & 0 & 0 & 0 & B & 0 & 0 & 0 & 0 \\
\hline D.M.B. & 0 & 0 & 0 & 0 & 0 & 0 & 0 & 0 & 0 \\
\hline B.G.F. & B & B & 0 & 0 & 0 & 0 & 0 & B & 0 \\
\hline P.M.A.P. & B & 0 & 0 & B & B & 0 & $R$ & $R$ & B \\
\hline J.J.D. & B & 0 & 0 & 0 & B & B & B & B & 0 \\
\hline L.A.R. & 0 & 0 & 0 & 0 & 0 & 0 & 0 & 0 & 0 \\
\hline L.E.V.L. & 0 & 0 & 0 & 0 & 0 & 0 & 0 & 0 & 0 \\
\hline F.G.J. & 0 & 0 & 0 & 0 & 0 & 0 & 0 & 0 & 0 \\
\hline L.C.B.S. & B & 0 & B & 0 & $R$ & B & B & B & B \\
\hline M.A.M. & 0 & 0 & 0 & 0 & 0 & 0 & 0 & 0 & 0 \\
\hline P.P.P. & 0 & 0 & 0 & 0 & 0 & 0 & 0 & 0 & 0 \\
\hline J.P.S. & B & 0 & 0 & 0 & B & 0 & B & B & B \\
\hline J.N.R.B. & B & 0 & 0 & 0 & B & 0 & 0 & 0 & 0 \\
\hline M.B.J. & 0 & 0 & 0 & 0 & 0 & 0 & 0 & 0 & 0 \\
\hline F.B.J. & B & 0 & 0 & 0 & B & 0 & 0 & B & 0 \\
\hline M.B.M. & 0 & 0 & 0 & 0 & 0 & 0 & 0 & 0 & 0 \\
\hline D.R.R.L. & B & 0 & 0 & 0 & B & 0 & 0 & B & 0 \\
\hline R.N.S. & 0 & B & B & B & B & 0 & 0 & 0 & 0 \\
\hline M.S. & 0 & B & 0 & 0 & 0 & 0 & 0 & 0 & 0 \\
\hline D.S.K. & 0 & 0 & 0 & 0 & 0 & 0 & 0 & 0 & 0 \\
\hline J.R.S. & 0 & 0 & 0 & B & 0 & 0 & 0 & 0 & 0 \\
\hline G.V.B. & 0 & 0 & 0 & 0 & 0 & 0 & 0 & 0 & 0 \\
\hline
\end{tabular}

LISTA DE ABREVIATURAS GRUPO A - CIRURGIÕES CARDIOVASCULARES COM PARTICIPAÇÃO ATIVA

E.B. Enio Buffolo; J.W.N. José Wanderley Netto; D.M.B. Domingo Marcolino Braile; B.G.F. Bayard Gontijo Filho; P.M.A.P. Pablo Maria Alberto Pomerantzeff; J.J.D. Jarbas Jakson Dinkhuysen; L.A.R. Luiz Antonio Rivetti; L.E.V.L. Luiz Eduardo Villaça Leão; F.G.J. Francisco Gregori Junior; L.C.B.S. Luiz Carlos Bento de Souza; M.A.M. Miguel Angel Maluf; P.P.P. Paulo Paredes Paulista; J.P.S. José Pedro da Silva; J.N.R.B. João Nelson Rodrigues Branco; M.B.J. Marcelo Biscegli Jatene -F.B.J. Fabio Biscegli Jatene; M.B.M. Miguel Barbero Marcial; D.R.R.L. Danton Richlin da Rocha Loures; R.N.S. Ricardo Nilson Sgarbieri; M.S. Max Schaldach; D.S.K. Decio Sylvestre Kormann; J.R.S. João Ricardo Santana; G.V.B. Gilberto Venossi Barbosa

\begin{tabular}{lccccccccccc}
\hline RESPOSTAS & $Q_{1}$ & $Q_{2}$ & $Q_{3}$ & $Q_{4}$ & $Q_{5}$ & $Q_{6}$ & $Q_{7}$ & $Q_{8}$ & $Q_{9}$ & TOTAL & $\%$ \\
\hline 0 & 14 & 20 & 21 & 20 & 13 & 21 & 18 & 16 & 20 & 163 & 78.7 \\
B & 9 & 3 & 2 & 3 & 9 & 2 & 4 & 6 & 3 & 41 & 19.8 \\
R & 0 & 0 & 0 & 0 & 1 & 0 & 1 & 1 & 0 & 3 & 1.5 \\
$\mathrm{r}$ & 0 & 0 & 0 & 0 & 0 & 0 & 0 & 0 & 0 & 0 & 0.0 \\
\hline TOTAL & 23 & 23 & 23 & 23 & 23 & 23 & 23 & 23 & 23 & 207 & 100.0 \\
\hline
\end{tabular}


ANDRADE, J. C. S. - Nova metodologia para ensino e ensaio de técnicas operatórias em cirurgia cardíaca. Rev. Bras. Cir. Cardiovasc., $9(1): 1-21,1994$.

TABELA 12

RESPOSTAS: O(ÓTIMO), B(BOM), R(REGULAR) E R(RUIM) PARA OS 9 QUESITOS FORMULADOS (Q, A $\left.Q_{\vartheta}\right)$ SEGUNDO O GRUPO P CONSTITUIDOO POR 30 CIRURGIÕES CARDIOVASCULARES COM PARTICIPAÇÃO PASSIVA (ASSISTENTES) NO LABORATÓRIO DE CIRURGIA.

\begin{tabular}{|c|c|c|c|c|c|c|c|c|c|}
\hline AVALIADOR & $Q_{1}$ & $Q_{2}$ & $Q_{3}$ & $Q_{4}$ & $Q_{5}$ & $Q_{6}$ & $Q_{7}$ & $Q_{8}$ & $Q_{9}$ \\
\hline J.H.P.F. & 0 & 0 & 0 & 0 & B & 0 & 0 & 0 & 0 \\
\hline H.V. & 0 & B & 0 & 0 & 0 & B & 0 & 0 & 0 \\
\hline R.C. & 0 & B & 0 & 0 & B & 0 & 0 & 0 & 0 \\
\hline R.G.R.C. & 0 & B & 0 & 0 & 0 & 0 & 0 & 0 & 0 \\
\hline J.I.V.A. & 0 & B & 0 & 0 & 0 & 0 & 0 & 0 & 0 \\
\hline L.F.A. & 0 & 0 & 0 & 0 & 0 & 0 & 0 & 0 & 0 \\
\hline M.G & 0 & 0 & 0 & B & B & 0 & 0 & 0 & 0 \\
\hline G.C. & 0 & B & 0 & 0 & 0 & 0 & 0 & 0 & 0 \\
\hline C.M.O. & B & 0 & 0 & 0 & B & 0 & 0 & 0 & 0 \\
\hline C.A.T. & 0 & B & 0 & 0 & B & 0 & 0 & 0 & 0 \\
\hline G.F.V. & 0 & B & 0 & 0 & 0 & 0 & 0 & 0 & 0 \\
\hline M.A.A. & 0 & 0 & 0 & 0 & 0 & 0 & 0 & 0 & 0 \\
\hline R.M. & 0 & 0 & 0 & 0 & 0 & 0 & 0 & 0 & 0 \\
\hline C.B.H. & 0 & 0 & 0 & 0 & 0 & 0 & 0 & 0 & 0 \\
\hline J.L.V. & 0 & 0 & 0 & 0 & 0 & 0 & 0 & 0 & 0 \\
\hline E.G.D.J. & 0 & 0 & 0 & 0 & 0 & 0 & 0 & 0 & 0 \\
\hline P.C.B. & 0 & 0 & 0 & 0 & 0 & 0 & 0 & 0 & 0 \\
\hline M.z. & 0 & 0 & 0 & 0 & 0 & 0 & 0 & 0 & 0 \\
\hline J.C.F.L. & 0 & 0 & 0 & 0 & 0 & 0 & 0 & 0 & 0 \\
\hline M.J.F.S. & 0 & 0 & 0 & 0 & 0 & 0 & 0 & 0 & 0 \\
\hline M.R.C. & 0 & B & 0 & 0 & 0 & 0 & 0 & B & 0 \\
\hline J.T.M. & 0 & 0 & 0 & 0 & 0 & 0 & 0 & 0 & 0 \\
\hline P.L.M. & 0 & 0 & 0 & 0 & B & 0 & $\mathbf{R}$ & 0 & 0 \\
\hline L.H.D. & B & B & B & B & 0 & 0 & 0 & 0 & 0 \\
\hline J.F.S. & 0 & 0 & 0 & 0 & 0 & 0 & B & B & 0 \\
\hline E.K.S. & B & 0 & 0 & 0 & 0 & 0 & B & 0 & 0 \\
\hline E.R. & 0 & 0 & 0 & 0 & 0 & 0 & 0 & 0 & 0 \\
\hline C.O.C. & 0 & 0 & 0 & 0 & 0 & 0 & 0 & 0 & 0 \\
\hline W.J.C. & 0 & 0 & 0 & 0 & 0 & 0 & 0 & 0 & 0 \\
\hline F.A.L. & 0 & 0 & 0 & 0 & 0 & 0 & B & 0 & 0 \\
\hline
\end{tabular}

LISTA DE ABREVIATURAS GRUPO P - CIRURGIÕES CARDIOVASCULARES COM PARTICIPAÇĀO PASSIVA J.H.P.F. José Honório Palma da Fonseca; H.V. Herminio Vega; R.C. Roberto Catani; R.G.R.C. Ruy Guilherme Rodrigues Cal; J.I.V.A. Jaime Ignacio Jaramillo Altamirano; L.F.A. LucianoFigueiredo Aguiar; M.G. Marcelo Grandini; G.C. Guido Caputti; C.M.O. Carlos Maximiliano Oliveira; C.A.T. Carlos Alberto Teles; G.F.V. Guilherme Flora Vargas; M.A.A. Mario Angelo Angeloni; R.M. Ricardo Mustafá; C.B.H. Carlos Borsato Herrera; J.L.V. José Luiz Verde; E.G.D.J. Eliud Garcia Duarte Júnior; P.C.B. Paulo César Biaggi; M.Z. Marcos Zaiantchick; J.C.F.L. João Carlos Ferreira Leal; M.J.F.S. Marcelo José Ferreira Soares; M.R.C. Marcos Ramos Carvalho; J.T.M. José Teles de Mendonça; P.L.M. Paulo Lavanieri Moreno; L.H.D. Luis H. Dussin; J.F.S. Jair F. Saadi; E.K.S. Eduardo K. Saadi; E.R. Edson Roswaz; C. O.C. Celso Otaviano Cordeiro; W.J.C. Wilson José Couto; F.A.L. Fernando Antonio Lucchesi

\begin{tabular}{lccccccccccc}
\hline RESPOSTAS & $Q_{1}$ & $Q_{2}$ & $Q_{3}$ & $Q_{4}$ & $Q_{5}$ & $Q_{6}$ & $Q_{7}$ & $Q_{8}$ & $Q_{9}$ & TOTAL & $\%$ \\
\hline O & 27 & 21 & 29 & 28 & 24 & 29 & 26 & 28 & 30 & 242 & 89.6 \\
B & 3 & 9 & 1 & 2 & 6 & 1 & 3 & 2 & 0 & 27 & 10.0 \\
R & 0 & 0 & 0 & 0 & 0 & 0 & 1 & 0 & 0 & 1 & 0.4 \\
$\mathrm{r}$ & 0 & 0 & 0 & 0 & 0 & 0 & 0 & 0 & 0 & 0 & 0.0 \\
TOTAL & 30 & 30 & 30 & 30 & 30 & 30 & 30 & 30 & 30 & 270 & 100.0 \\
\hline
\end{tabular}


ANDRADE, J. C. S. - Nova metodologia para ensino e ensaio de técnicas operatórias em cirurgia cardiaca. Rev. Bras. Cir. Cardiovasc., $9(1): 1-21,1994$.

TABELA 13

RESPOSTAS: O(ÓTIMO), B(BOM), R(REGULAR) E R(RUIM) PARA OS 9 QUESITOS FORMULADOS (Q, A Q $)$ SEGUNDO O GRUPO G CONSTITUIDDO POR 40 CIRURGIÕES DE OUTRAS ESPECIALIDADES (CIRURGIA GERAL) QUE ASSISTIRAM À EXPOSIÇÃO EXPLICATIVA DO MÉTODO E A SEGMENTOS DOS "VIDEO TAPES" DOS LABORATÓRIOS DE CIRURGIA.

\begin{tabular}{|c|c|c|c|c|c|c|c|c|c|}
\hline AVAL IADOR & $Q_{1}$ & $\mathrm{Q}_{2}$ & $Q_{3}$ & $Q_{4}$ & $Q_{5}$ & $Q_{6}$ & $Q_{7}$ & $Q_{8}$ & $\overline{Q_{9}}$ \\
\hline S.G. & 0 & 0 & 0 & 0 & 0 & 0 & 0 & 0 & $\mathrm{O}$ \\
\hline M.O.T. & B & B & B & B & B & $R$ & B & B & B \\
\hline R.F.B.C & B & B & B & B & B & B & B & B & B \\
\hline A.P.K. & B & B & B & B & B & B & B & B & B \\
\hline A.C.D. & 0 & 0 & 0 & 0 & 0 & 0 & 0 & 0 & 0 \\
\hline D.G.S. & B & B & B & B & B & B & B & B & B \\
\hline V.J.S. & B & 0 & 0 & B & B & 0 & $R$ & 0 & 0 \\
\hline L.R.N. & 0 & B & B & 0 & 0 & 0 & 0 & 0 & 0 \\
\hline M.D.S.G. & B & B & B & 0 & 0 & 0 & B & B & 0 \\
\hline A.A.S. & 0 & B & 0 & 0 & B & 0 & 0 & 0 & $\mathrm{O}$ \\
\hline J.Q.S.L. & 0 & 0 & $B$ & 0 & B & 0 & 0 & 0 & O \\
\hline E.K.T. & 0 & 0 & 0 & 0 & 0 & 0 & 0 & 0 & 0 \\
\hline A.S.C.J. & B & B & B & B & B & 0 & 0 & 0 & $\mathrm{O}$ \\
\hline J.R.F. & B & 0 & B & B & 0 & 0 & 0 & 0 & 0 \\
\hline F.A.G.G. & 0 & 0 & 0 & 0 & 0 & B & 0 & 0 & 0 \\
\hline R.L.C. & B & B & 0 & 0 & 0 & 0 & 0 & 0 & 0 \\
\hline A.L.S. & B & 0 & 0 & B & 0 & B & 0 & B & B \\
\hline P.N.S. & 0 & B & B & B & B & B & 0 & B & B \\
\hline D.I.J. & B & 0 & B & 0 & 0 & 0 & 0 & 0 & $\mathrm{O}$ \\
\hline G.S.S. & B & B & B & B & B & B & B & B & B \\
\hline S.N. & B & B & B & B & B & B & B & B & B \\
\hline O.R.R. & B & 0 & B & 0 & 0 & 0 & B & 0 & 0 \\
\hline Z.M.C. & 0 & B & B & B & 0 & 0 & 0 & 0 & 0 \\
\hline C.O.B.J. & 0 & B & B & 0 & 0 & 0 & 0 & 0 & O \\
\hline M.I.F. & 0 & 0 & 0 & B & 0 & B & 0 & 0 & 0 \\
\hline F.P.L. & B & B & B & 0 & B & B & 0 & 0 & B \\
\hline M.L.L.P. & B & $B$ & B & B & 0 & 0 & 0 & B & B \\
\hline R.H.I. & B & 0 & B & 0 & B & 0 & 0 & B & 0 \\
\hline L.S.L. & B & 0 & B & 0 & B & 0 & 0 & 0 & 0 \\
\hline R.M.A. & B & 0 & B & B & 0 & B & 0 & 0 & 0 \\
\hline E.M. & 0 & 0 & 0 & 0 & 0 & 0 & 0 & 0 & 0 \\
\hline M.D.P. & B & 0 & 0 & 0 & 0 & B & 0 & 0 & \\
\hline C.A.P.L. & B & B & 0 & 0 & B & 0 & 0 & 0 & 0 \\
\hline E.P.S. & B & 0 & B & B & B & 0 & B & B & B \\
\hline L.H.G.S. & 0 & 0 & 0 & 0 & 0 & 0 & 0 & 0 & 0 \\
\hline O.F.J. & B & B & 0 & 0 & B & B & 0 & 0 & B \\
\hline J.G.A.C. & B & B & B & 0 & 0 & B & B & 0 & \\
\hline L.H.A.C. & 0 & 0 & 0 & B & B & B & B & B & B \\
\hline M.S.V.X. & 0 & 0 & 0 & 0 & B & 0 & 0 & 0 & 0 \\
\hline B.S.L. & B & B & B & B & 0 & 0 & 0 & 0 & 0 \\
\hline
\end{tabular}

LISTA DE ABREVIATURAS GRUPO G - CIRURGIŌES DE OUTRAS ESPECIALIDADES

S.G. Saul Goldenberg; M.O.T. Murched Omar Taha; R.F.B.C. Regina de Faria Bittencourt Costa; A.P.K. Andréa Paula Kafejian; A.C.D. Antonio Carlos Donoso; D.G.S.Domingos Guerino Silva; V.J.S.Venilton José Siqueira; L.R.N. Luiz Roberto do Nascimento; M.D.S.G. Marli Donoti S. Garcia;A.A.S. Arnold Adolph Steger; J.Q.S.L. Jovelino Quintino Souza Leăo; E.K.T. Edson Kiyoschi Tsunematsu; A.S.C.J. Alfredo Soares Cabral Júnior; J.R.F.Jefferson Rago Ferreira; F.A.G.G. Fernando Augusto Garcia Guimarāes; R.L.C. Rubens Luis Costa; A.L.S. Adriano Leite Soares; P.N.S. Paulo Nogueira Saad; D.I.J.Décio landoli Júnior; G.S.S. Gilmar Silva Salvador; S.N. Syllene Nunes; O.R.R. Olavo Ribeiro Rodrigues; Z.M.C. Zuleika Monteiro de Carvalho; C.O.B.J. Claudio Oliveira Borba Júnior; M.I.F. Mario Indolfo Filho; F.P.L. Fabio Piovezan Lyra; M.L.L.P. Maria Ligia Lyra Pereira; R.H.I. Roberto Hiroschi leiri; L.S.L. Lino Sanchez Laranjeira; R.M.A. Rosangela M. Araújo; E.M. Evaldo Marchi; M.D.P. Max Domingues Pereira; C.A.P.L. Cirilo Antonio de Paula Lima; E.P.S. Edivar Pereira dos Santos; L.H.G.S. Luiz Humberto Garcia de Souza; O.F.J. Octacilio Felicio Júnior; J.G.A.C. José Geraldo A. Calomeno; L.H.A.C. Luiz Henrique A. Calomeno; M.S.V.X. Mario Sérgio Viana Xavier, B.S.L. Braulio Souza Lessa

\begin{tabular}{lccccccccccc}
\hline RESPOSTAS & $\mathrm{Q}_{1}$ & $\mathrm{Q}_{2}$ & $\mathrm{Q}_{3}$ & $\mathrm{Q}_{4}$ & $\mathrm{Q}_{5}$ & $\mathrm{Q}_{6}$ & $\mathrm{Q}_{7}$ & $\mathrm{Q}_{8}$ & $\mathrm{Q}_{9}$ & TOTAL & $\%$ \\
\hline $\mathrm{O}$ & 15 & 20 & 16 & 22 & 21 & 24 & 28 & 27 & 26 & 199 & 55.3 \\
$\mathrm{~B}$ & 25 & 20 & 24 & 18 & 19 & 15 & 11 & 13 & 14 & 159 & 44.2 \\
$\mathrm{R}$ & 0 & 0 & 0 & 0 & 0 & 1 & 1 & 0 & 0 & 2 & 0.5 \\
$\mathrm{r}$ & 0 & 0 & 0 & 0 & 0 & 0 & 0 & 0 & 0 & 0 & 0.0 \\
TOTAL & 40 & 40 & 40 & 40 & 40 & 40 & 40 & 40 & 40 & 360 & 100.0 \\
\hline
\end{tabular}


ANDRADE, J. C. S. - Nova metodologia para ensino e ensaio de técnicas operatórias em cirurgia cardíaca. Rev. Bras. Cir. Cardiovasc., 9 (1): 1-21, 1994.

\section{COMENTÁRIOS}

A idéia do Laboratório de Cirurgia nasceu na Escola Paulista de Medicina com a finalidade de apresentar em peças anatômicas, detalhes de técnicas cirúrgicas e discuti-las entre um cirurgião experiente e pequeno grupo de cirurgiões residentes ou estagiários. A limitação do campo de observação e do número de observadores fez com que se incorporasse ao método de ensino o sistema de projeção de vídeo em "telão". Com a disponibilidade desse recurso e com a certeza de que se a peça permanecesse estável nos seus pontos naturais de fixação, assemelhando-se à sua posição anatômica, se melhoraria a capacidade de transmissão dos ensinamentos construiu-se o suporte apresentado (Figura 3). A utilização na sua confecção de fios de alumínio como hastes de fixação para as veias cavas, artéria pulmonar e aorta, assim como, de fixação com tração elástica das veias pulmonares, permitiu a maleabilidade desejada dessas estruturas anatômicas durante o manuseio cirúrgico.

A opção de se trabalhar com corações humanos, apesar das naturais dificuldades de obtenção, deve-se ao fato de que os corações de animais (cães, ovelhas, porcos, bois etc), apesar de suas semelhanças, apresentam importantes diferenças anatômicas, dificultando a "realidade" pretendida.

O fato de se trabalhar com corações sem nenhum processo de fixação ou conservação (exceto o frio) faz com que eles se apresentem com características de cor, consistência, textura e aspecto semelhantes ao que o cirurgião costuma observar nos corações sob cardioplegia. É esta a impressão que se quer obter do cirurgião quando se the oferece uma "operação" no Laboratório de Cirurgia Cardíaca. Com as veias cavas e a aorta canuladas e com conexão ao sistema de C.E.C., a aorta pinçada e o coração flácido, a impressão é realmente de um coração parado por cardioplegia hipotérmica. Como as peças são conservadas sob congelação, apresentam um natural escurecimento de suas superfícies. Após a descongelação, através da hidratação por solução de sulfito de sódio a $2 \%$, consegue-se um reavivamento da coloração. Isto, acrescido da maleabilidade proporcionada pelo suporte, confere à "operação" uma impressão de realidade. Por outro lado, não é desproposital toda a encenação armada no Laboratório. Sua montagem reeditando um Centro Cirúrgico, desde a aparelhagem instrumental até toda a vestimenta apropriada, tem por finalidade incutir na mente da equipe operatória a sensação de um real ambiente cirúrgico e as reações e emoções de um verdadeiro ato operatório. Atingidos esses objetivos, já se está preparando, moldando o comportamento do pessoal médico e paramédico dentro de uma sala operatória, cum- prindo, portanto, uma função educativa (GOFFI ${ }^{3}$, GOLDENBERG \& BEVILACQUA ${ }^{4}$ )

A realização da "operação" por cirurgião escoIhido, competente, habilidoso e didático, por si só, já conferiria êxito ao procedimento. A gravação e projeção simultânea em tela de grande dimensão $(4.0 \times 3.0 \mathrm{~m})$ permite que se ofereça o aprendizado a um maior número de pessoas. Estas, na dependência da "operação" podem ser residentes e estagiários em princípio de formação (nas operações simples, habituais), ou profissionais já formados (nas operações complexas, técnicas recentes ou novas proposições).

A projeção, nesta metodologia, ganha muito em nitidez e visão de detalhes, proporcionados pela ausência de sangue no campo e maior liberdade dada ao "camera-men" já que sua atuação não confere nenhum risco de contaminação ao paciente. Obtém-se assim uma visão quase perfeita das estruturas envolvidas.

Por outro lado, o fato de não existir o habitual risco de vida do paciente, de uma cirurgia real, deixa o cirurgião também à vontade para: repetir uma manobra não entendida, expor pontos anatômicos importantes, mostrar uma variante técnica ou tática, esclarecer dúvidas etc, o que, evidentemente, também enriquece o potencial de ensino do método.

Sua apresentação inicial durante o XI Simpósio Internacional Unicór, em julho de 1993 e suas reedições em novembro de 1993, durante o 3 ํ Congresso da Sociedade de Cirurgia Cardiovascular do Estado de São Paulo e em março de 1994, durante - 21으 Congresso Nacional de Cirurgia Cardíaca cativaram a comunidade participante como muito bem expressam algumas frases retiradas das opiniões dos cirurgiões e comentadores:

“...das mais modernas técnicas de ensino da cirurgia cardíaca";

"Como subproduto... as gravações em vídeo.. (constituem) material didático de inestimável valor";

“...que todos os eventos das Sociedades de Cirurgia Cardíaca tenham esta nova modalidade de ensino integrada ao programa de forma definitiva";

“..é uma idéia original e criativa, a maior contribuição ao ensino da Técnica Operatória em Cirurgia Cardíaca de nossa época";

“...cirurgiões mais jovens ensaiem com facilidade técnicas complexas que dificilmente poderiam executar com segurança sem treinamento prévio";

"...trouxe inovações importantes na comunicação entre os cirurgiōes";

“...já se consagrou em nosso meio e deverá ser 
ANDRADE, J. C. S. - Nova metodologia para ensino e ensaio de técnicas operatórias em cirurgia cardiaca. Rev. Bras. Cir. Cardiovasc., 9 (1): 1-21, 1994.

integrada sistematicamente a todos os Congressos de nossa especialidade";

“...também deverá ser utilizado nos Centros de Formação de cirurgiōes cardiovasculares...";

“...conseguindo elevar o nivel técnico de nossos cirurgiōes";

"...com as vantagens de magnificar a imagem, transmitir para a platéia, responder a todas as questões, esclarecer dúvidas, repetir gestos, manobras ou mudanças de técnica, despreocupando-se com o tempo, hemostasia e resultado final da operação";

“...é uma valiosa contribuição ao ensino, aprendizado e discussão de técnicas cirúrgicas, revolucionando a maneira de apresentaçāo";

"...tem se mostrado excelente, fazendo com que - Laboratório de cirurgia atinja completamente os objetivos propostos...";

"...metodologia revolucionária para o ensino da cirurgia...";

"...excelente contribuição para o desenvolvimento da cirurgia cardíaca brasileira...";

"...revolucionando a maneira de apresentação dos detalhes técnicos de cada tipo de intervenção...";

"Acreditamos que esta nova modalidade deverá revolucionar a apresentação e o ensino da cirurgia nos próximos anos...";

"...é sem dúvida uma novidade didática $e$ instrutiva que veio para ficar...";

"... simular de uma forma perfeita a maioria dos procedi-mentos cirúrgicos...";

"... um dos pontos importantes do médoto utilizado é de cunho didático...";

"... tipo de metodologia possa e deva ser utilizado na formação de cirurgiōes cardíacos jovens...";

“... que o método seja divulgado em outros países onde com certeza causará grande interesse.";

"O método proposto é ótimo, por propiciar ao cirurgião um modelo em que pode praticar e demonstrar (também ensinar) as técnicas de correção cirúrgica.";

"A idéia é original e perfeitamente apropriada para o ensino de técnicas e táticas operatórias...";

"... e organizamos um grupo de trabalho, no qual utilizamos suas idéias, pretendendo estabelecer atividade idêntica como rotina.";

"O método é um meio excelente para a demonstração cirúrgica e para o aprendizado, em qualquer nível: acadêmicos, residentes e profissionais da especialidade.";

“... concluo pelo real valor desta experiência que, sem dúvida alguma, vem acrescentar ao ensino da cirurgia cardíaca mais um importante avanço.";

"... conheci o método proposto e considerei avanço excepcional para o ensino e treinamento em cirurgia cardiaca.";

"... veio preencher uma lacuna que dificultava o ensino em cirurgia cardíaca.";

"... é audacioso, inovador, moderno, didático e inteligente...";

"O método é de validade inquestionável...".

Apesar de estas manifestações atribuírem um excelente conceito a esta nova metodologia de ensino, procurou-se quantificar esses conceitos através da análise dos questionários. Estes, respondidos pelos três grupos de cirurgiōes documentaram, sem margem a dúvida, a aceitação da metodologia.

Com o questionário constituído por 9 quesitos e distribuido a um total de 93 avaliadores: 23 do Grupo A, 30 do Grupo P e 40 do Grupo G, foi obtido um total de 837 respostas, ou seja: 207 no Grupo A, 270 no Grupo B e 360 no Grupo G.

Tomou-se como critério de aceitação, de aprovação da Nova metodologia para ensino e ensaio de técnicas operatórias em cirurgia cardíaca, apenas as respostas ótimo e bom. Verifica-se que no Grupo A, das 207 respostas, obteve-se 163 $(78.7 \%)$ ótimo e $41(19.8 \%)$ bom, ou seja: $98.5 \%$ de aprovação; no Grupo P com 270 respostas, 242 $(89.6 \%)$ foram ótimo e $27(10 \%)$ foram bom, num total de aceitação de $99.6 \%$; no Grupo G, das 360 respostas, obteve-se 199 (55.3\%) ótimo e 159 $(44.2 \%)$ bom, perfazendo $99.5 \%$ de aceite.

Somadas as respostas dos três grupos obtevese: $604(72.2 \%)$ ótimo, $227(27.1 \%)$ bom, $6(0.7 \%)$ regular e $0(0 \%)$ ruim, o que entre ótimo e bom confere à Nova metodologia para o ensino e ensaio de técnicas operatórias em cirurgia cardíaca uma aceitação de $99.3 \%$

O teste do Qui quadrado utilizado para o total das respostas, com o objetivo de comparar os grupos de cirurgiões em relação às respostas, mostrou uma associação significante do Grupo P, ou seja, de Cirurgiões cardiovasculares assistentes do Laboratório, com a resposta ótimo. Essa alta porcentagem $(89.6 \%)$ de ótimo evidenciou que o método satisfez amplamente aqueles que o presenciaram. A porcentagem de ótimo também foi consideravelmente alta $(78.7 \%)$ no Grupo A, ou seja, de cirurgiões cardiovasculares com participação ativa no Laboratório de Cirurgia.

Apesar dessa porcentagem de ótimo ter sido menor no Grupo G, de cirurgiões de outras espe- 
ANDRADE, J. C. S. - Nova metodologia para ensino e ensaio de técnicas operatórias em cirurgia cardiaca. Rev. Bras. Cir. Cardiovasc., 9 (1): 1-21, 1994.

cialidades, ela ainda foi alta considerando que este Grupo não assistiu ao Laboratório e sim à uma exposição explicativa do método.

A comparação das respostas de aprovação (ótimo e bom) com as de não aprovação (regular e ruim) mostrou diferenças extremamente grandes, não justificando análise estatística.

Essa grande aceitação do método nos três eventos realizados parece configurar, conforme também opinião de vários participantes dos Laboratórios de Cirurgia realizados, um lugar de destaque no ensino da técnica operatória em cirurgia cardiaca, doravante, pelo menos nos congressos da especialidade. Neles, dado o grande número de participantes, diluem-se o esforço e o custo do empreendimento. Nessa situação, o emprego do método objetivará mais o ensino da técnica do que seu treinamento.

Já sua utilização em Serviços de Cirurgia Cardíaca, notadamente em Escolas Médicas, terá naturalmente uma função de treinamento, de aperfeiçoamento técnico. Nessa situação, é evidente que o método pode ser simplificado, dispensando-se toda a encenação até agora empregada, como também a gravação e transmissão de vídeo, resumindo-se a montagem à simulação da caixa torácica. Isto implicaria em diminuição dos custos, acrescida de menor demanda de tempo. É o que está sendo organizado, este ano, para o treinamento de residentes e estagiários da Disciplina de Cirurgia Cardiovascular da Escola Paulista de Medicina.

Apesar de o método com a utilização de corações normais permitir a realização de técnicas empregadas para correção de cardiopatias congênitas, nem todas serão passíveis de reprodução Como é óbvio, algumas operações têm correção baseada no próprio defeito (ex: Tetralogia de Fallot, Comunicação interventricular, Transposição dos grandes vasos da base, "Atrio ventricularis communis" etc). Assim sendo, necessitar-se-ia organizar um banco de peças com defeitos congênitos, o que evidentemente obriga a uma conexão com um Serviço de Verificação de Óbitos ou de Anatomia Patológica para busca e armazenamento dessas peças.

É vantajoso que o armazenamento dessas peças, com defeitos congênitos ou não, em congelador, seja feito com as mesmas montadas no suporte, a fim de evitar a necessidade de dois descongelamentos (um para a montagem e outro para a demonstração). O procedimento com um único descongelamento, como já se constatou, proporciona melhor aspecto à peça quando de sua utilização no Laboratório de Cirurgia.

A alternativa do descongelamento único com montagem imediata tem o inconveniente da neces- sidade de tempo para esse mister, o que a torna quase inviável nos eventos com várias operações consecutivas.

Dados os contatos e interesses demonstrados por cirurgiōes e professores de cirurgia de várias especialidades, uma outra bastante viável possibilidade desta metodologia de ensino e/ou treinamento é sua aplicação em outras áreas de cirurgia.

O progresso da Informática e suas múltiplas aplicaçōes sugerem a possibilidade de poder-se dispor futuramente de um "paciente operável" em computador. Assim, esse "soft" desejável permitiria a "dissecção eletrônica" ou seja, à medida que o cirurgião traçasse as linhas de incisão, apareceriam os planos subjacentes e suas estruturas. A programação poderia inclusive determinar o acerto ou não da técnica empregada pelo "cirurgião".

Apesar da viabilidade dessa possibilidade eletrônica, o entusiástico interesse que o Laboratório de Cirurgia tem despertado e sua pronta viabilidade, parece determinar em futuro imediato um grande incremento na sua utilização.

\section{CONCLUSÕES}

\section{A metodologia apresentada permite:}

1) perfeita reprodutividade de atos operatórios em ambientes coletivos;

2) excelente imagem dos procedimentos através da projeção em telão, com uma visão total e detalhada do campo cirúrgico;

3) aumento na capacidade de transmissão dos ensinamentos devido à limpeza e clareza do campo operatório bem como a descontração da equipe cirúrgica, determinadas pela ausência de sangue e de risco operatório ou de contaminação do "paciente";

4) interrupção da "operação" a qualquer instante e sem risco algum para esclarecimento técnico ou tático, repetição de manobra, explicação mais minuciosa ou mesmo discussão com os participantes;

5) formação de material didático de uso permanente, pelo registro em "video tape" das "operações";

6) realização dessas "operações" em cardiopatias congênitas ou adquiridas, através da criação de um arquivo de peças anatômicas com defeitos congênitos ou adquiridos, congeladas;

7) antevisão da extensão desse procedimento a outras especialidades. 
ANDRADE, J. C. S. - Nova metodologia para ensino e ensaio de técnicas operatórias em cirurgia cardiaca. Rev. Bras. Cir. Cardiovasc., 9 (1): 1-21, 1994.

RBCCV $44205-227$

ANDRADE, J. C. S. - A new methodology for teaching and rehearsing of operative tecniques in cardiac surgery. Rev. Bras. Cir. Cardiovasc., 9 (1): 1-21, 1994.

ABSTRACT: Utilizing equipment and conditions similar to the operating room we have reproduced all that it is necessary for simulating cardiac surgery with extracorporeal circulation. Surgeries are performed in a dummy who has a freshly fixed human anatomic piece firmly attached to a device specially developed and which is placed inside the thorax of the dummy. The procedures performed are videotaped and are also displayed simultaneously in a large screen situated in an auditorium with free communication with the surgical team. The system was utilized in July 1993 - XI Unicor International Symposium, and in November $1993-3^{\text {rd }}$ SCICVESP Congress and in March $1994-21^{\text {th }}$ Brazilian Congress of Cardiac Surgery with the following procedures being perfomed: Senning, Jatene, cavo-pulmonary anastomosis, Konno, Monaghian, mitral valve replacement, labyrinth surgery, retrograde cardioplegy, implantable defibrillator and orthotopic heart transplantation. The method was received enthusiastically by our colleagues and proved to be an efficient teaching technique as well as an excellent rehearsal tool due to its: 1) perfect reproducibility of the surgical conditions; 2) excellent image of the procedures as viewed in the large screen at the auditorium; 3) improvement in the way surgery is learned and taught due to the fact that the surgical field is clear cut and the surgical team is relaxed; 4) interruption of the surgical act without the complications that could develop during a "real" surgery; "surgery" can be interrupted at any time; 5) obtaining a video library with high quality definition images of different procedures; 6 ) we can foresee a frozen archive of hearts with congenital or acquired heart defects prepared to undergo surgical correction and 7) the future utilization of this technique in other surgical specialties.

DESCRIPTORS: heart surgery, methods; surgery, operative methods; cardiac surgery, teaching.

\section{Agradecimentos}

Ao Prof. Dr. Costabile Gallucci (in memorian), de quem em vida tive o privilégio de ser aluno, amigo, companheiro, "filho" e beneficiário de imensas lições de grandeza d'alma.

Ao Prof. Dr. Vicente Forte, de quem absorvi os primeiros ensinamentos em cirurgia torácica e cardiovascular e cujo entusiástico trabalho me prendeu à especialidade, pelo estímulo e apoio presentes, desde 0 início até os dias de hoje.

Ao Prof. Dr. Enio Buffolo, companheiro e amigo, do banco escolar à atividade profissional, pela amizade e convívio prolífico durante todos esses anos e pelo estímulo permanente para a realização deste trabalho.

Ao Prof. Dr. Boris Barone, amigo e companheiro (colega "veterano"), que me indicou e abriu o caminho da especialidade, pelo carinho e amizade fraterna.

Ao Prof. Dr. João Nelson R. Branco, ao Dr. Guido Caputti e à Srta. Maria Aparecida Stendardi da Costa, pela participação fundamental no preparo e montagem dos vários Laboratórios de Cirurgia.

Ao Prof. Dr. Sigmar Horst Cardoso e à Disciplina de Patologia Médica da E.P.M., pela sempre presente e imprescindível colaboração.
Aos Profs. Drs. Antonio Carlos C. Carvalho, Luciano Figueiredo Aguiar e Carlos Alberto Teles, companheiros de trabalho, pelo apoio e colaboração no desenvolvimento do projeto.

Aos amigos e companheiros da Disciplina de Cirurgia Cardiovascular, professores, pós-graduandos, residentes, enfermeiras, instrumentadoras, por todo o apoio e incentivo.

Aos funcionários do Hospital São Paulo, Hospital Unicór e da Cardiocir pelo apoio e auxílio na elaboração dos Laboratórios.

Aos "operadores" do Laboratório, pela habilidade, competência e didática, registradas na videoteca, que foram o fator de sucesso da metodologia: Domingos M. Braile, Marcelo B. Jatene, Francisco Gregori Júnior, Bayard Gontijo Filho, Miguel A. Maluf, Max Schaldach, José Wanderley Neto, Luiz Carlos Bento de Souza, Fábio B. Jatene, Décio S. Kormann, Pablo M. A. Pomerantzeff, Luiz A. Rivetti, Ricardo N. Sgarbieri, Jarbas J. Dinkhuysen, Paulo Paredes Paulista, Miguel Barbero Marcial, João Ricardo Santana, Luiz Eduardo Villaça Leão, Danton R. da Rocha Loures, Enio Buffolo e José Pedro da Silva.

Aos Profs. Dr. Neil Ferreira Novo e Dra. Iara Juliano, da Disciplina de Bioestatística do Departamento de Medicina Preventiva, pela prestimosa e imprescindível análise estatística deste trabalho e, 
ANDRADE, J. C. S. - Nova metodologia para ensino e ensaio de técnicas operatórias em cirurgia cardiaca. Rev. Bras. Cir. Cardiovasc., 9 (1): 1-21, 1994

muito mais, pela sempre pronta e dedicada atenção.

Ao Dr. Ruy Guilherme Rodrigues Cal, amigo solicito, a cujo conhecimento de Informática devo a materilização deste trabalho, pelo despreendimento, dedicação e competência demonstrados durante todo o preparo e edição do texto.

Ao Sr. Leopoldo Amaral Sales Filho, da Vídeo 33 , pela qualidade profissional e pela dedicação amiga e prestimosa.

Aos Srs. Carlos Alberto de Assis e Sérgio Trevelin, da SC Produções, pela dedicação e pela valiosa documentação fotográfica.

À Sita. Eliane Rodrigues Junqueira, pelo trabalho dedicado durante a digitação do texto.

À Braile Biomédica, pela colaboração efetiva e entusiasta desde os passos iniciais do projeto.

À Ethicon - Divisão de Produtos Profissionais da Johnson \& Johnson, através dos Srs. Mario Ferradoza e Ricardo Pereira, e das Sras. Suely Augusto, Fernanda Fernandes e Nanci Ferreira, pelos esforços e o indispensável apoio econômico para a realização do projeto e para a impressão desta publicação.

Aos cadáveres que anonima mas imprescindivelmente, cederam parte de seus corpos, o meu respeito e reconhecimento.

\section{REFERÊNCIAS BIBLIOGRÁFICAS}

1 CARREL, A. - On the experimental surgery of the thoracic aorta and the heart. Ann. Surg. 52: 83-95, 1910.

2 CONFORTI, V. L. P. \& MAGALHÃES, H. P. - História da cirurgia. In: MAGALHĀES, H. P. Técnica cirúrgica e cirurgia experimental. São Paulo. Sarvier, 1983, p.1-4.

3 GOFFI, S. S. - Técnica cirúrgica: bases anatômicas, fisiopatológicas e técnicas da cirurgia. Rio de Janeiro. São Paulo, 1978, vol I:494 p.

4 GOLDENBERG, S. \& BEVILACQUA, R. G. - Bases da cirurgia. Sāo Paulo. E.P.U. Editora da U.S.P., 1981, $304 \mathrm{p}$.

5 LYONS, A. S. \& PETRUCELLI, R. J. - Medicine: an illustrate history. New York, Harry N. Abrans Inc., $1978,616 \mathrm{p}$.

6 MAGALHÃES, H.P. - Técnica cirúrgica e cirurgia experimental. São Paulo. Sarvier, 1983, 338 p.

7 SIEGEL, S. - Estatística no paramétrica. México, Trillas, 1975, $346 \mathrm{p}$.

8 OLIVEIRA, A.B. - A evolução da medicina até o inicio do século $X X$. Săo Paulo, Pioneira: Secretaria de Estado da Cultura, 1981, 434 p. 